\title{
Detailed Modeling of Microwave Energy Deposition in EBT Devices
}

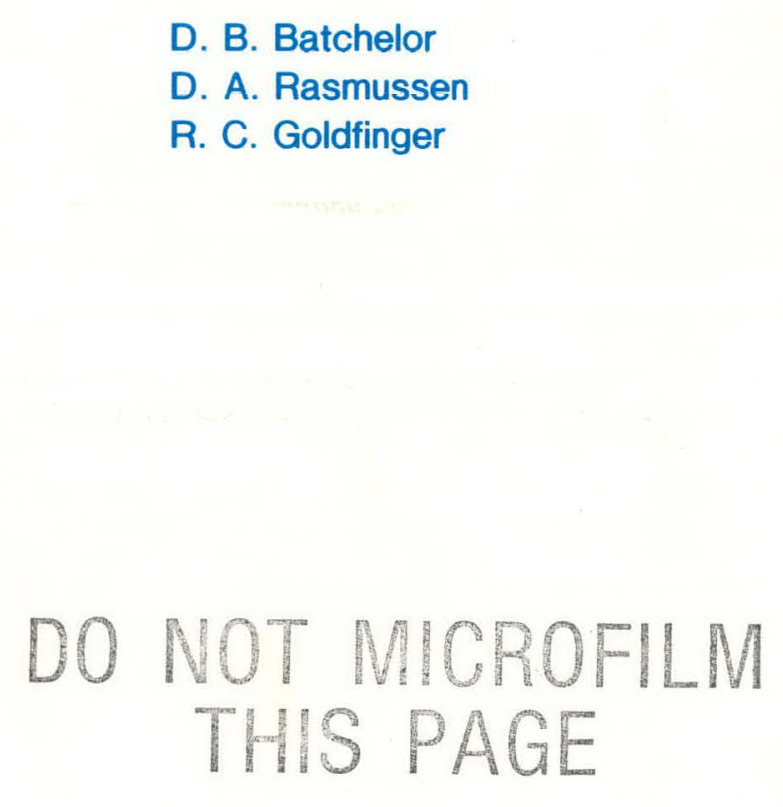

UNION CARBIDE

D. B. Batchelor

D. A. Rasmussen

R. C. Goldfinger 


\section{DISCLAIMER}

This report was prepared as an account of work sponsored by an agency of the United States Government. Neither the United States Government nor any agency Thereof, nor any of their employees, makes any warranty, express or implied, or assumes any legal liability or responsibility for the accuracy, completeness, or usefulness of any information, apparatus, product, or process disclosed, or represents that its use would not infringe privately owned rights. Reference herein to any specific commercial product, process, or service by trade name, trademark, manufacturer, or otherwise does not necessarily constitute or imply its endorsement, recommendation, or favoring by the United States Government or any agency thereof. The views and opinions of authors expressed herein do not necessarily state or reflect those of the United States Government or any agency thereof. 


\section{DISCLAIMER}

Portions of this document may be illegible in electronic image products. Images are produced from the best available original document. 


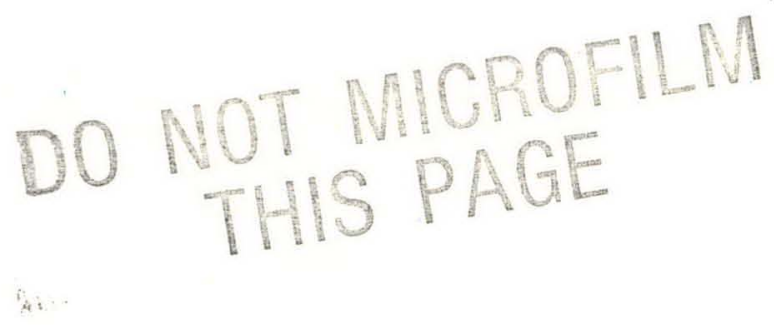

Printed in the United States of America. Available from National Technical Information Service

U.S. Department of Commerce

5285 Port Royal Road, Springfield, Virginia 22161

NTIS price codes-Printed Copy: A03; Microfiche A01

This report was prepared as an account of work sponsored by an agency of the United States Government. Neither the U nited States Government nor any agency thereof, nor any of their employees, makes any warranty, express or implied, or assumes any legal liability or responsibility for the accuracy, completeness, or usefulness of any information, apparatus, product, or process disclosed, or represents that its use would not infringe privately owned rights. Reference herein to any specific commercial product, process, or service by trade name, trademark, manufacturer, or otherwise, does not necessarily constitute or imply its endorsement, recommendation, or favoring by the United States Government or any agency thereof. The views and opinions of authors expressed herein do not necessarily state or reflect those of the United States Government or any agency thereof. 


\title{
DETAILED MODELING OF MICROWAVE ENERGY DEPOSITION IN EBT, DEVICES
}

\author{
D. B. Batchelor \\ D. A. Rasmussen
}

Fusion Energy Division

R. C. Goldfinger

UCC-ND Computer Sciences

Date Published - August 1983

Prepared by the

OAK RIDGE NATIONAL LABORATORY

Oak Ridge, Tennessee 37830

operated by

UNION CARBIDE CORPORATION

for the

U.S. DEPARTMENT OF ENERGY

under contract $W$-7405-eng-26

ORNL/TM-8770

Dist. Category UC-20 


\section{DISCLAIMER}

This report was prepared as an account of work sponsored by an agency of the United States Government. Neither the United States Guvernment nor any agency thereof, nor any of their employees, makes any warranty, express or implied, or assumes any legal liability or responsibility for the accuracy, completeness, or usefulness of any information, apparatus, product, or process disclosed, or represents that its use would not infringe privately owned rights. Reference herein to any specific commercial product, process, or service by trade name, trademark, manufacturer; or otherwise does not necessarily constitute or imply its endorsement, rewommendation, or favoring by the United 3tates Government or any agonoy thoreof. The viowe aud npinions of authnrs expressed berein do not necessarily state or reflect those of the United States Government or any agency thereof. 


\section{CONTENTS}

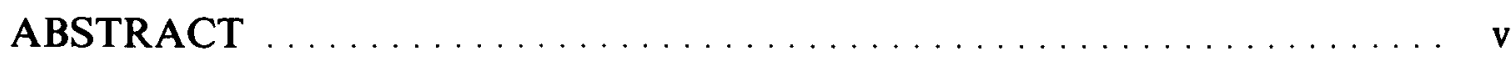

I. INTRODUCTION $\ldots \ldots \ldots \ldots \ldots \ldots \ldots \ldots \ldots \ldots \ldots \ldots \ldots$

II. EQUILIBRIUM MODEL $\ldots \ldots \ldots \ldots \ldots \ldots \ldots \ldots \ldots \ldots \ldots$

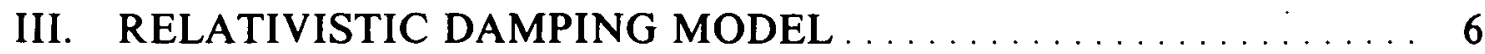

IV. RAY-TRACING RESULTS FOR SINGLE-PASS

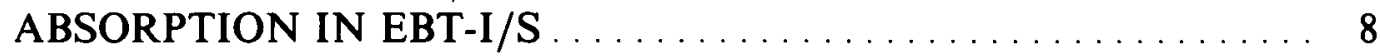

V. POWER DEPOSITION IN EBT-1/S USING THE POWER BALANCE MODEL . . . . . . . . . . . . . . . . . . . . 11

VI. EXPERIMENTAL MEASUREMENTS $\ldots \ldots \ldots \ldots \ldots \ldots \ldots \ldots$

VII. PROJECTIONS FOR EBT-P . . . . . . . . . . . . . . . . . . . . 19

VIII. CONCLUSIONS .............................. 23

Appendix A. EQUATIONS FOR THE POWER BALANCE

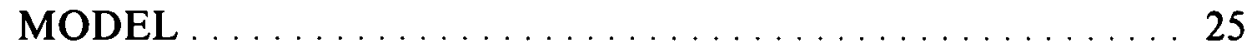

REFERENCES . . . . . . . . . . . . . . . . . . . . . . 28 


\begin{abstract}
Ray-tracing studies have been combined with a simple wave power balance model to provide a complete, albeit approximate, description of microwave power deposition in the ELMO Bumpy Torus (EBT-I), EBT-Scale (EBT-S), and EBT Proof-of-Principle (EBT-P) devices. Electron cyclotron absorption of ordinary and extraordinary waves by the combined core plasma and relativistic annuli is calculated using a fully relativistic damping package developed for the RAYS geometrical optics code. The rays are traced in finite-beta bumpy cylinder plasma equilibria that are obtained from the Oak Ridge National Laboratory (ORNL) two-dimensional (2-D) equilibrium code. These results for direct, single-pass absorption are combined with results from a statistical model for the deposition of multiply reflected and mode-converted waves to obtain estimates of the power deposited in the core, surface, and annulus plasma components. Wave absorption by the annuli and by the core components at the fundamental and second harmonic resonances, reflection and Budden tunneling of the extraordinary mode at the right-hand cutoff, and conversion between ordinary and extraordinary modes upon wall reflection are the processes included in the power balance model. Experimental measurements of wave power flux on the cavity wall in EBT-S made with a simple microwave calorimeter are in good agreement with predictions of the model for a variety of operating configurations.
\end{abstract}




\section{INTRODUCTION}

In this paper we present detailed numerical calculations of the microwave power deposition in the various plasma components of ELMO Bumpy Torus (EBT) devices. Previous studies using the RAYS geometrical optics code ${ }^{1-3}$ have concentrated on power deposition in the core and surface plasma components. The picture that emerged was one of weakly damped rays making many transits across the device, with wall reflections and repeated ordinary-extraordinary mode conversions playing an important role in the ultimate energy deposition. In order to deal with the complicated, essentially random nature of the wave propagation after a few wall reflections, a simple zerodimensional (0-D) power balance model was developed that treated the sources, sinks, and conversion properties of the ordinary and extraordinary modes in a globally averaged way. ${ }^{4}$ Wave absorption by the annulus was included in the power balance model empirically by using measured estimates of the fraction of power going to the rings. Recently, we have developed a fully relativistic damping package for the RAYS code that involves no expansion in $T_{e} / m c^{2}$ and allows an arbitrary number of cyclotron harmonics to be included. ${ }^{5}$ Combining ray-tracing studies with the power balance model, we are now able to theoretically treat the complete power deposition problem. A great many simplifying approximations are, of course, still necessary to make progress.

Our ray-tracing calculations are carried out using a bumpy cylinder equilibrium with parameters chosen to model as closely as posible the plasma configurations of EBT/EBT-Scale (EBTI/EBT-S) or EBT Proof-of-Principle (EBT-P). Initially, we trace through the plasma an ensemble of rays that are launched at varying angles from a point at the midplane near the waveguide opening. The power carried by each ray is weighted by a suitable antenna radiation pattern, and the power deposited in each plasma component for a single pass through the device is determined by integrating over a solid angle. Typically, we find that only a small fraction of the total injected power is absorbed in a single pass. Hence, the power remaining after a single pass is spread across the wall surface and greatly randomized with respect to direction of propagation. The deposition of this power can be calculated using the power balance model. In the end, we obtain estimates for the total power deposited in the core, annulus, and surface plasma components. These three plasma components are defined as shown on the left of Fig. 1. The distinguished magnetic field line which serves as the boundary between the core and surface components is that field line which is tangential to the vacuum second harmonic resonance at the midplane. It is here that the peak of the annulus pressure tends to form. The core plasma is magnetohydrodynamic (MHD) stabilized by the annulus and neoclassically confined, whereas the surface plasma is unstable and confinement is very poor. The standard EBT heating geometry is illustrated on the right of Fig. 1 showing fundamental and second harmonic cyclotron resonances and the right-hand cutoff for the extraordinary mode.

It is pussible with these models to calculate some experimentally measurable quantities. In particular, one can calculate the total microwave power flux on the cavity wall under various configurations with the microwave feed in the cavity of interest or adjacent cavities turned on or off. Measurements of the wall power flux made with a microwave calorimeter are in good agreement with theoretical predictions for a wide range of operating conditions. This confirms the calculated rate of global power absorption (or cavity $Q$ ).

Another important application of the models presented here is to make projections for larger, hotter, and denser devices such as the proposed EBT-P experiment. One must recognize at the outset that the results are sensitive to assumed features of the plasma equilibrium such as density, temperature, profile shapes, and details of the distribution function. Since these assumptions are based upon rough scaling laws rather than upon concrete experimental data, as the EBT-I calculations are, the results are necessarily speculative. However, these calculations are useful as a first step in quantifying the considerable differences to be found in heating advanced devices. We find that absorption at the second harmonic resonance by the core plasma component, although negligible in EBT-I/S, is crucial in EBT-P. Similarly, ordinary mode absorption at the fundamental resonance may bc important. Also, linig absurption is predicted by linear theory to be much weaker in EBT-P than in EBT-I/S. 


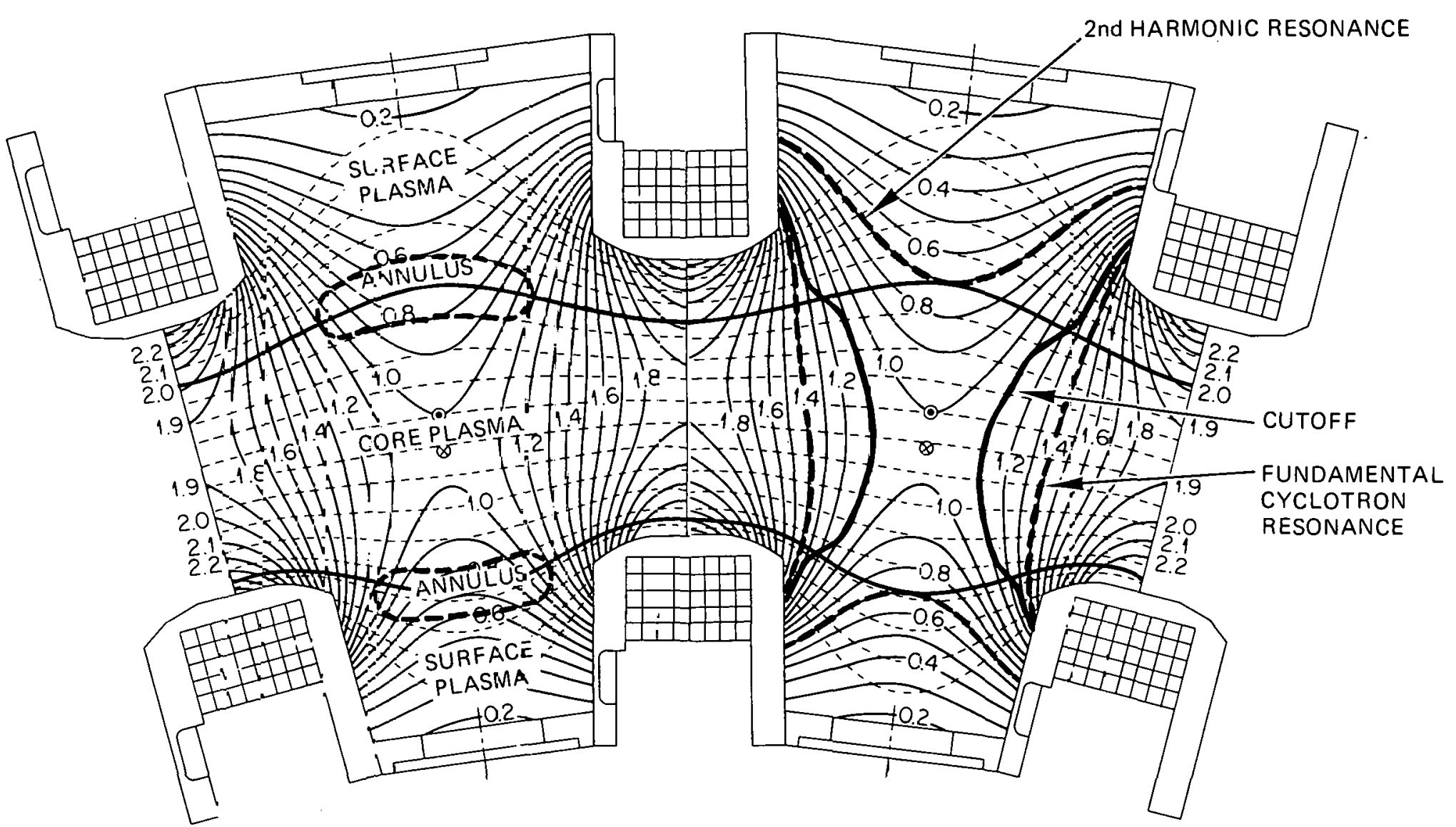

Fig. 1. Equatorial crose section of tro EBT-S cavities Shown are magnetic field lines (dashed); mod-B contours (solid); core, annulus, and surface plasma components; and the location of tie fundament al resonance, the right-hand cuto:f, and the second barmonic resonance. 


\section{EQUILIBRIUM MODEL}

The finite-beta magnetic fields and annulus density profile are calculated using a twodimensional (2-D) tensor pressure MHD equilibrium code, ${ }^{6} \underline{B}(r, z)=\underline{\nabla} \psi \times \underline{\sim} \Theta$, where $\psi$ satisfies

$$
\nabla^{*}+\underset{\sim}{\nabla} \ln \sigma \cdot \underset{\sim}{\psi}=-\frac{r^{2}}{\sigma} \frac{\partial P_{\|}}{\sigma \psi}
$$

and

$$
\begin{aligned}
& \sigma=1+\frac{P_{\perp}-P_{\|}}{B^{2}}, \\
& P_{\|}(B, \psi)=\hat{P}_{\|}(B) g(\psi)+P(\psi), \\
& P_{\perp}(B, \psi)=\hat{P}_{\perp}(B) g(\psi)+P(\psi), \\
& \hat{P}_{\|}(B)=\frac{S}{2}\left(B_{c}-B\right)^{2} \text { for } B \leqslant B_{c}, \\
& \hat{P}_{\perp}(B)=\frac{S}{2}\left(B_{c}^{2}-B^{2}\right) \text { for } B<B_{c}, \\
& \hat{P}_{\|}=\hat{P}_{\perp}=0 \text { for } B \geqslant B_{c}, \\
& S=\text { annulus strength factor related to beta , } \\
& B_{c}=\text { cutoff field related to annulus length, } \\
& g(\psi)=\text { cubic polynomial which vanishes outside } \psi_{1} \leqslant \psi \leqslant \psi_{2} \text { and determines radial } \\
& \quad \text { extent of annulus . }
\end{aligned}
$$

The coefficients of $g(\psi)$ are adjusted to give an annulus extending from $r=r_{\min }$ to $r=r_{\max }$ with the annulus pressure peak at $r=r_{\text {peak }}$. The core density is chosen to be a function of $\psi(x)$ alone, which is nearly flat inside the annulus and decreases through the annulus to a constant level $n_{m}$ in the surface plasma:

$$
n_{c}(\underset{\sim}{x})=n_{c}[\psi(x)]=\frac{1+c-\tanh \left[\psi(\underset{\sim}{x})-\psi_{o}\right] / \delta^{2}}{1+c+\tanh \left(\psi_{o} / \delta^{2}\right)},
$$

where

$$
\begin{aligned}
\psi_{o} & =\psi\left(r_{\text {peak }}, 0\right) \\
c & =n_{\infty} /\left(1-n_{\infty}\right)\left[1+\tanh \left(\psi / \delta^{2}\right)\right], \\
\delta & =\left(r_{\max }-r_{\min }\right) / 2 .
\end{aligned}
$$


The core temperature is taken to be proportional to the core density,

$$
T_{c}(\underset{\sim}{x})=T_{e}\left[\frac{n_{c}(\underset{x}{x})}{n_{o}}\right]^{1 / 2} .
$$

The parameters of the equilibrium were chosen so as to model as closely as possible specific mid-Tmode experimental runs for which fairly complete data were available. ${ }^{7}$ For the EBT-I case chosen, hard x-ray data give the annulus temperature $T_{A}=145 \mathrm{keV}$ and an average density of $n_{A}=$ $1.8 \times 10^{11} \mathrm{~cm}^{-3}$. The diamagnetic signal gives an annulus stored energy of approximately $8 \mathrm{~J}$ per ring. The parameters of the pressure function $g(\psi)$ were adjusted to place $r_{\text {peak }}$ at $13.8 \mathrm{~cm}$, approximately the location of the vacuum second harmonic resonance for the nominal EBT-I toroidal field coil operating current of $5 \times 10^{3} \mathrm{~A} . \quad B_{r}$ is adjusted to give a half-pressure annulus length of about $11 \mathrm{~cm}$. The parameter $S$ is fixed so as to give $\beta=0.042$, where

$$
\beta \equiv \frac{8 \pi P_{\perp}\left(r_{\max }, z=0\right)}{B(0,0)^{2}}
$$

This corresponds to local $\beta_{\text {local }}=8 \pi P_{\perp}\left(r_{\max }, 0\right) / B^{2}\left(r_{\max }, 0\right)=0.102$. The externally imposed vacuum bumpy cylinder field is due to filamentary current loops spaced $20 \mathrm{~cm}$ from the midplane with a radius necessary to produce a $2: 1$ mirror ratio on axis.

The equilibrium code provides $P_{\|}$and $P_{\perp}$ as functions of position. On the other hand, the relativistic damping model assumes an isotropic Maxwellian distribution function and requires density $n_{A}(x)$ and temperature $T_{A}(x)$ as independent functions. For the purposes of wave damping we have adopted a simple model in which $T_{A}$ is constant and $n_{A} \propto P=P_{\perp}+P_{\|}$:

$$
n_{A}(x)=n_{A}(B, \psi)=\frac{\beta B^{2}(0,0)}{8 \pi T_{, A}} \frac{P(B, \psi)}{P_{\max }}
$$

Using $T_{A}=145 \mathrm{keV}, \beta=0.042$ gives a peak annulus density of $2 \times 10^{11} \mathrm{~cm}^{-3}$. When $n_{A}(x) T_{A}$ is integrated over the volume of the annulus, one obtains a total stored energy of $\omega_{\perp}=8.6 \widetilde{\mathrm{J}}$, in good agreement with the experimental value. Interferometry and soft $x$-ray data give for the core plasma $n_{o}=4.7 \times 10^{11} \mathrm{~cm}^{-3}$ and $T_{e}=440 \mathrm{eV}$. These EBT-I parameters and those for EBT-S are summarized in Table $I$.

Figure 2 shows midplane profiles $(z=0)$ of core density $\omega_{p e}^{2}(r) / \omega^{2}$, annulus density $\omega_{p A}^{2}(r) / \omega^{2}$, and magnetic field $\Omega_{e}(r) / \omega$, where $\omega_{p j}=\left(4 \pi e^{2} n_{j} / m_{j}\right)^{1 / 2}=$ plasma frequency for the $j$ th component, $\Omega_{e}=e B / m_{e} c=$ electron cyclotron frequency, and $\omega / 2 \pi=18 \mathrm{GHz}=$ EBT-I microwave frequency. One can see that for the low value of beta used for EBT-I the depression of the magnetic field at the annulus location is minimal. Nevertheless, these plasma parameters do represent the characteristic T-mode of operation.

Figure 3 shows a cross section of the bumpy cylinder model for EBT-S used in these calculations. Shown are the finite-beta magnetic field lines, the fundamental and second harmonic cyclotron resonances, the extraordinary mode cutoff, and contours of the annulus density. 
Table I. Plasma parameters used in ray-tracing calculations.

\begin{tabular}{llll}
\hline & \multicolumn{1}{c}{ EBT-I } & \multicolumn{1}{c}{ EBT-S } & \multicolumn{1}{c}{ EBT-P } \\
\hline Ring temperature, $T_{A}(\mathrm{keV})$ & 145 & 390 & $1 \times 10^{3}$ \\
Ring density, $n_{A}\left(\mathrm{~cm}^{-3}\right)$ & $1.8 \times 10^{11}$ & $5 \times 10^{11}$ & $7 \times 10^{11}$ \\
Ring peak radius, $r_{\text {peak }}(\mathrm{cm})$ & 13.8 & 12.2 & 19 \\
& & & \\
Ring beta $\left(\frac{8 \pi \mathrm{T}_{\mathrm{A}} \mathrm{n}_{\mathrm{A}}}{B^{2}}\right)^{r_{\text {prak }}}, \beta$ & 0.116 & 0.312 & 0.16 \\
& & & \\
Ring stored energy, $W_{\perp}$ & $8.5 \mathrm{~J} / \mathrm{ring}$ & $37 \mathrm{~J} / \mathrm{ring}$ & \\
Peak core density, $n_{o}$ & $5 \times 10^{11}$ & $10^{12}$ & $1.1 \times 10^{13}$ \\
Peak core temperature, $T_{e}(\mathrm{eV})$ & 400 & 500 & $2 \times 10^{3}$ \\
Microwave power, $P_{\mu}(\mathrm{kW})$ & 40 & 175 & $1 \times 10^{3}$ \\
Fill pressure, $P_{o}$ (torr) & $5 \times 10^{-6}$ & $8 \times 10^{-6}$ & \\
\hline
\end{tabular}

ORNL-DWG 83-2615 FED
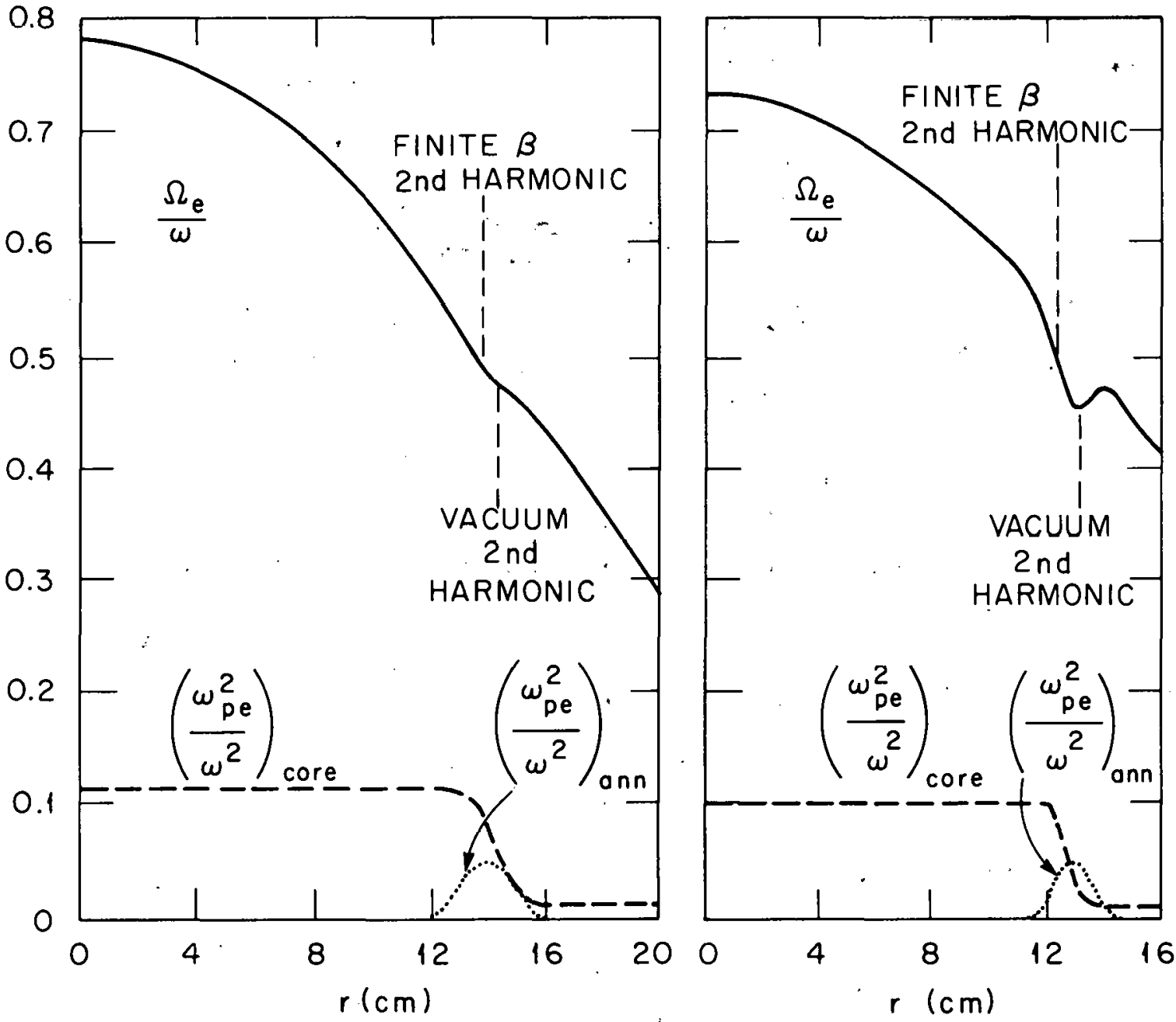

Fig. 2. Midplane profiles of core density $\left(\omega_{p e}^{2} / \omega^{2}\right)$, annulus density $\left(\omega_{P A}^{2} / \omega^{2}\right)$, and magnetic field $\Omega_{e}(r) / \omega$ for (a) the EBT-I equilibrium and (b) the EBT-S equilibrium used in the ray tracing. 


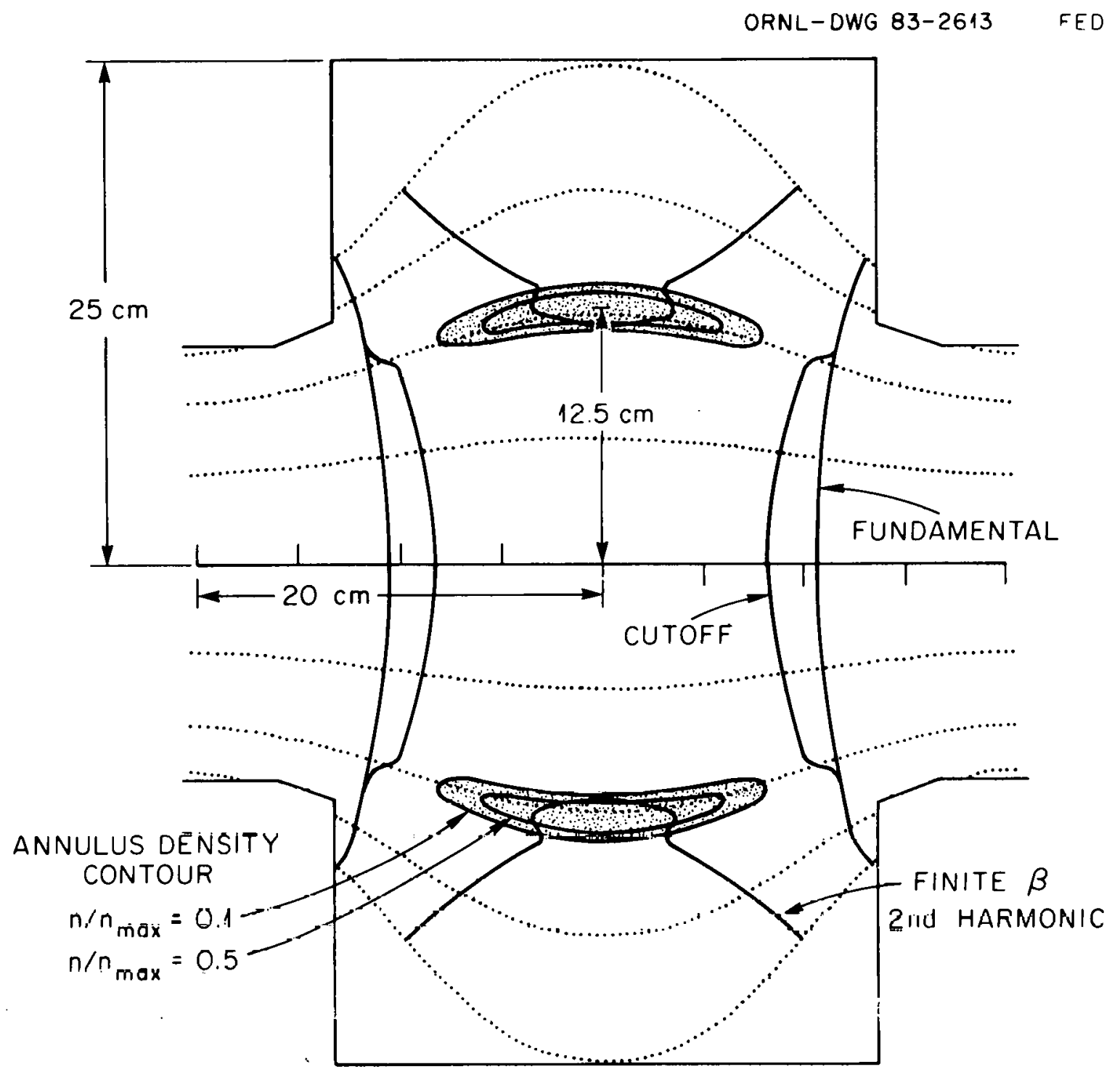

Fig. 3. Cross section of the bumpy cylinder equilibrium in EBT-S used in ray tracing.

\section{RELATIVISTIC DAMPING MODEL}

In our calculations absorption of the microwaves by the annulus and by the core component at the second harmonic resonance is calculated using Poynting's theorem:

$$
\underset{d S}{d \underline{d}}|\underline{S}|=\frac{4 \pi}{c} \underset{\sim}{E^{*}} \cdot \underset{\sigma^{H}}{=} \underline{\sim}
$$

Here, $s$ is the arc length along a ray, $S=\operatorname{Re}\left[E^{*} \times(n \times E)\right]=$ Poynting's $\left.\left.E\right)\right]=$ Poynting's vector, $\underline{n}=c k / \omega=$ real refractive index, and $\underset{\sigma^{H}}{=}=$ Hermitian part of the relativistic conductivity 
tensor. The real refractive index is determined from the ray-tracing code, which uses the cold plasma dispersion relation

$$
D(\underline{n})=\operatorname{det}(\underset{\approx}{D})=\operatorname{det}\left[\left(1-n^{2}\right) \underset{\approx}{I}+\underline{n} \boldsymbol{n}+\frac{4 \pi_{1}}{\omega} \underset{\sigma^{A}}{\approx}\right]=0,
$$

where $\underset{\sigma^{A}}{=}=$ anti-Hermitian part of the plasma conductivity. Two plasma components are included in $\underset{\sigma^{A}}{\approx}$ : a cold core plasma component and an annulus component for which $\underset{\approx}{\sigma}$ is also assumed to be of the cold form. The electric field eigenvectors $\underset{E}{E}$ used in the equation are determined from the cold plasma dispersion tensor with both components included, $\underset{\approx}{D} \underline{E}=0$. It should be mentioned that this procedure gives accurate values for the damping rate near the second harmonic resonance but is not valid near the fundamental resonance because of finite $T_{e}$ modification to the eigenvectors.

In calculating $\underset{\approx}{\sigma^{H}}$ an isotropic relativistic Maxwellian distribution function is assumed:

$$
F(\underline{p})=\frac{1}{m^{3} c^{3}} \frac{\rho n_{A}}{4 \pi K_{2}(\rho)} \exp \left[-\rho\left(1+\frac{p \prod^{2}+p_{\perp}^{2}}{m^{2} c^{2}}\right)^{1 / 2}\right],
$$

where $\rho=m_{e} c^{2} / T_{A}$ and $K_{2}(\rho)$ is the modified Bessel function. An arbitrary number of cyclotron harmonics can be retained and all Bessel functions containing finite Larmor radius effects are included without expansion. Details of the calculation of $\underset{\sigma^{H}}{\approx}$ may be found in Ref. 5 .

As an example, Fig. 4 shows the behavior of $k_{i} \equiv \operatorname{Im}(k)$ as a function of $\Omega_{e} / \omega$ for various values of $T_{e}$. The calculation is for a frequency of $f_{\mu}=60 \tilde{\mathrm{GHz}}$, density $n_{e}=4.4 \times 10^{12} \mathrm{~cm}^{-3}$, and perpendicular propagation $n_{\|}=0$. Twenty cyclotron harmonics were included in $\underset{\sigma}{\sigma}$. The peaked cyclotron harmonic structure that is seen at low temperatures washes out at higher temperature as adjacent cyclotron resonances broaden and overlap. A crucial feature for EBT annulus calculations is that for fixed frequency $\Omega_{e} / \omega$ the damping rate peaks as a function of temperature at some finite value of $T_{e}$. For example, near the second harmonic resonance the maximum of $k_{\|}$ occurs at $T_{e} \sim 70-90 \mathrm{keV}$. This effect can be understood physically as follows: cyclotron harmonic absorption is a finite Larmor radius effect, $k_{\|} \sim\left\langle J_{l}\left(k_{\perp} v_{\perp} / \Omega\right)\right\rangle$, so that at low temperature $k_{i}$ increases with $k_{\perp} v_{\perp} / \Omega$. However, at high temperatures the distribution function is widely spread out in velocity space so that the density of particles resonant with a wave of given $k, \omega$ decreases with increasing temperature. In most cases it is this mechanism rather than the peaking of the Bessel function that produces the maximum in $k_{\|}$. As temperature increases and the cyclotron resonances broaden, the total absorption integrated over all frequencies continues to increase with temperature. This is the inverse, through Kirchhoff's law, of synchrotron radiation which increases with temperature. However, in a situation such as EBT annuli where the wave frequency is fixed and the relativistic particles exist only in a region $\Omega_{e} / \omega \sim 1 / 2$, the absorption for a wave with given $k$, $\omega$ decrease above about $100 \mathrm{keV}$.

Calculation of the absorption by the core plasma is a much simpler task. In the ray tracing code a nonrelativistic weak damping expansion is used that is valid in the Doppler regime. ${ }^{3}$ 


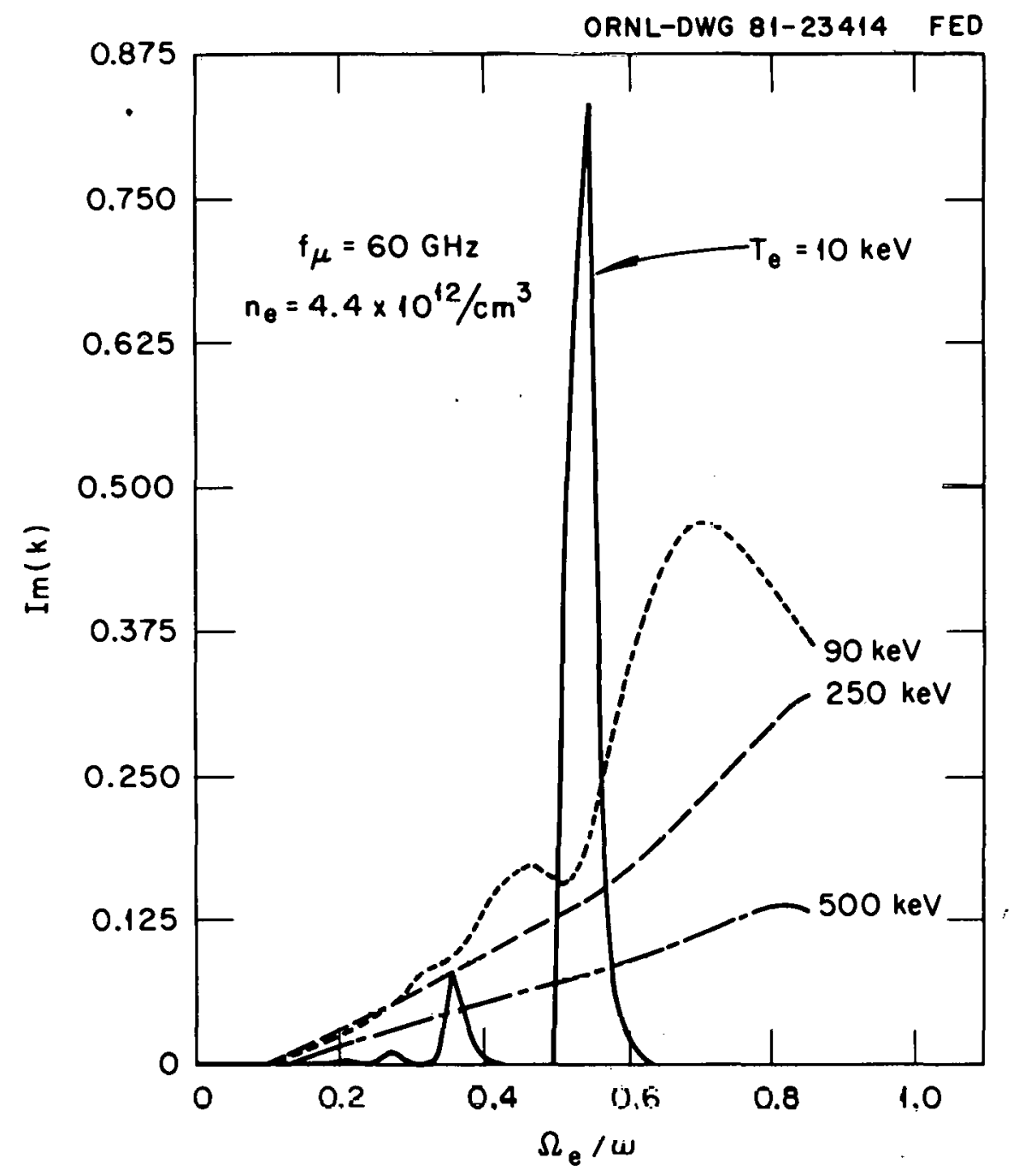

Fig. 4. Imaginary part of $k$ for extraordinary mode propagating perpendicular to $B$. Twenty cyclotron harmonics are included.

\section{RAY-TRACING RESULTS FOR SINGLE-PASS ABSORPTION IN EBT-I/S}

The microwaves in EBT are injected from waveguides lucialed at the midplanc of each mirror sector. In vider to calculate the dirubl puwer deposition in a single pass through the plasma, an ensemble of rays with various angles of propagation is traced from a point near the cavity wall representing the waveguide opening. The rays are followed until they emerge from the plasma at the cavity wall. For extraordinary mode rays, one or more reflections may occur in the plasma at the right-hand cutoff. The absorption is calculated on the basis of two plasma components, annulus and core, as described above. Three absorption prucesses are involvcd: (1) absorption by the annulus in the vicinity of the second harmonic resonance, (2) absorption by the core at the second harmonic, and (3) absorption by the core at the fundamental resonance. Since the fundamental resonance is inaccessible to the midplane-launched extraordinary mode, direct absorption at the fundamental occurs only for the ordinary mode. 
Figures 5(a) and 5(b) show typical ray paths in EBT-I for the extraordinary mode in the equatorial plane and midplane, respectively. In Fig. 5(a) the location of the annulus, the fundamental and finite-beta second harmonic resonance, and the right-hand cutoff are shown, and in Fig. 5(b) the intersection of the midplane with the annulus and the cavity wall is shown. Associated with each ray are two numbers $\left(f_{a n n}^{x}, f_{2 n d}^{x}\right)$, which indicate the fraction of power initially carried by the ray deposited in the annulus and in the core component at the second harmonic resonance, respectively. We see that $10-20 \%$ of a typical ray's energy is absorbed by the annulus in EBT-I, whereas. only a few percent is absorbed by the core component at the second harmonic resonance. The ordinary mode is much more weakly damped by both the annulus and core at the second harmonic resonance. Although the ordinary mode can propagate directly to the fundamental resonance, because of the low density and temperature of the core component in EBT-I, a maximum of a few percent of the energy carried by an ordinary mode ray is absorbed there.

ORNL-DWG $82-2340 \quad$ FED

(a)EQUATORIAL PLANE

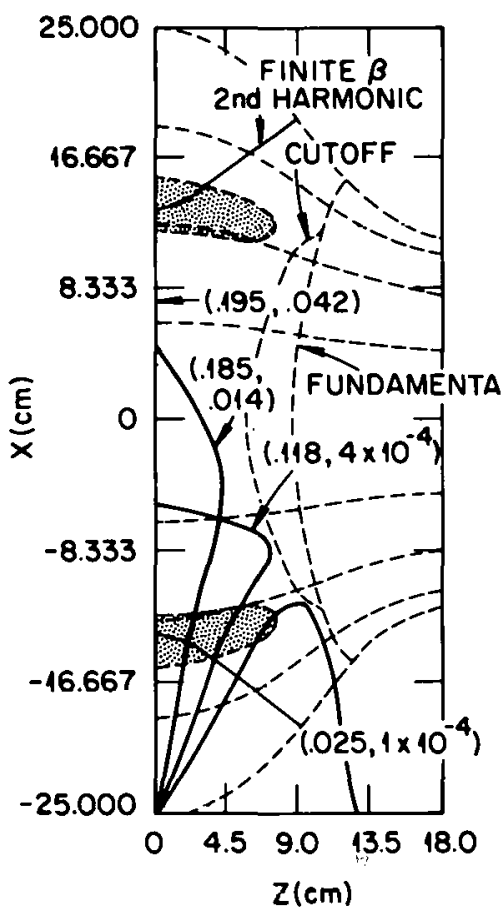

(b) MIDPLANE

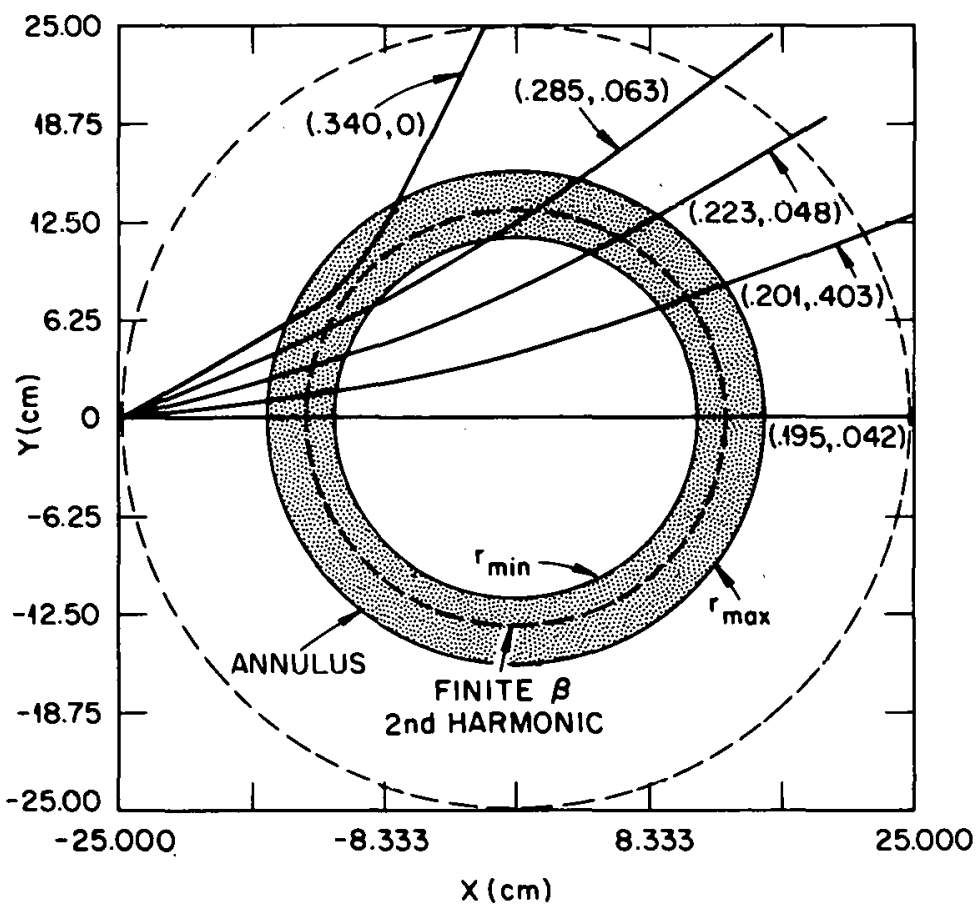

Fig. 5. Ray paths in EBT-I for extraordinary mode in (a) the equatorial plane and (b) the midplane. Numbers in parentheses are the fraction of power carried by the ray absorbed by the annulus component and by the core components at the second harmonic resonance, respectively.

To calculate the fraction of the total injected power $P_{i n j}$ deposited in each component we must integrate the angular distribution of launched power and the fractional power absorption over solid angle. In EBT-I and EBT-S the microwaves are launched from a waveguide mounted on the cavity wall without any directive antenna structurc. The radiation pattern is not measured and the difficulty of calculating the radiation pattern is compounded by the presence of the cavity wall and the plasma. For these calculations we have made the assumption that power is distributed in solid angle $d \Omega$ according to the law

$$
P d \Omega=\frac{3 P_{i n j}}{2 \pi} \cos ^{2} \theta d \Omega
$$


where $\theta$ is the angle with respect to the axis of the waveguide. This pattern is symmetric about the axis and gives total injected power $\boldsymbol{P}_{i n j}$ when integrated over the forward hemisphere. This pattern is similar to that obtained in the limit of an overmoded waveguide radiating into free space. The fraction \langle\rangle$_{j}^{i}$ of the injected power in mode $i$, deposited in plasma component $j$, is determined by integrating over solid angle

$$
\langle f\rangle_{j}^{i}=\frac{1}{P_{i n j}^{i}} \int d \phi d \theta \sin \theta f_{j}^{i}(\theta, \phi) p^{i}(\theta)
$$

where $f_{j}^{i}(\theta, \phi)$ is the fraction of power lost to that component by the $i$ mode ray launched in direction $\theta, \phi$ and $P_{i n j}^{i}$ is the total i mode power radiated.

The dominant mode waveguide used to launch the microwaves in EBT-I is oriented so as to predominantly excite the ordinary mode. We assume that the injected power $P_{i n j}$ is partitioned between the excited ordinary mode $P_{i n j}^{o}$ and the extraordinury mode $P_{i n j}^{x}, P_{i n j}=P_{i n j}^{x}+P_{i n j}^{o}$ and that the radiation pattern for both modes is $P(\theta)$. We have investigated the consequences of allowing $P_{i n j}^{o}$ to range from 0.5 to 0.9 and found that for EBT-I the effect is negligible. Therefore, for these calculations, the mix of modes excited is taken to be $P_{i n j}^{o}=P_{i n j}^{x}=0.5 P_{i n j}$.

Numerically carrying out the integral above, with $f(\theta, \phi)$ obtained from ray tracing, wc find for EBT-I that the fraction of extraordinary mode power deposited in the annulus and in the core component at the second harmonic resonance is

$$
\langle f\rangle_{a n n}^{x}=0.083 \quad\langle f\rangle_{2 n d}^{x}-0.01 \quad \text { (extraordinary mode) }
$$

respectively. For the ordinary mode at the annulus, the core plasma at the second harmonic, and the core plasma at the fundamental, we find

$$
\langle f\rangle_{a n n}^{o}=0.009\langle f\rangle_{2 n d}^{o}-0.0 \quad\langle f\rangle_{\text {ist }}^{o} \quad \text { (ordinary mode) }
$$

respectively. Assuming equal excitation of the ordinary and extraurdinary mode, $P_{i n j}^{x}=P_{i i j}^{u}=$ $P_{i n j} / 2$ gives for the single-pass power deposited in the annulus

$$
P_{a n n}=\langle f\rangle_{a n n}^{x} P_{i n j}^{x}+\langle f\rangle_{a n n}^{o} P_{i n j}^{o}=0.045 P_{i n j}
$$

and in the core at the second harmonic

$$
P_{2 n d}=\langle f\rangle_{2 n d}^{x} P_{i n j}^{x}+\langle f\rangle_{2 n d}^{o} P_{i n j}^{o}=0.005 P_{i n j}
$$

Only abuut $5 \%$ of the lutal injested power is absorber in one pass of the microwaves through the device. The remaining $0.455 P_{i n j}$ in the extraordinary mode and $0.49 P_{i n j}$ in the ordinary mude are spread widely around the surface of the cavity wall. In particular, by integrating over those rays that pass through the fundamental resonance, we find that $32 \%$ of the unabsorbed ordinary mode power is transmitted to the high field region. Absorption of the remaining power is discussed in the next section in terms of the puwer balancc model.

Similar numbers are obtained in the EBT-S calculations:

$$
\begin{array}{ll}
\langle f\rangle_{a n n}^{x}=0.045 & \langle f\rangle_{2 n d}^{x}=0.007 \\
\langle f\rangle_{a n n}^{o}=0.003 & \langle f\rangle_{2 n d}^{o}=0 \quad\langle f\rangle_{1 s t}^{o}=0.003
\end{array}
$$


Again, assuming a 50/50 ordinary/extraordinary excitation, we find that $0.97 P_{i n j}$ remains after one pass. The power absorbed by the ring is only $P_{a n n} \sim 0.025 P_{i n j}$, a smaller fraction than for EBT-I even though $W_{\perp}$ is four times larger. This is because of reduced ring absorptivity due to increased temperature.

\section{POWER DEPOSITION IN EBT-I/S USING THE POWER BALANCE MODEL}

For the present calculations the 0-D power balance model of Ref. 4 has been modified in several important ways. An improved ring absorption model has been developed in which the annulus absorptivity is estimated directly using the relativistic absorption code. An energy absorption channel for the core plasma component at the second harmonic resonance has been included. Although unimportant for EBT-I, absorption by the core component at the second harmonic is quite large for hotter, denser devices such as EBT-P. A third plasma region for the extraordinary mode has been introduced that allows us to model the relatively weaker power flux from the inside of the plasma when strong absorption occurs at the second harmonic resonance. Finally, the model has been reformulated such that additional sources and sinks can easily be incorporated. This makes it possible to calculate self-consistently the power coupled into the microwave calorimeter for various combinations of fed and unfed adjacent cavities.

For purposes of extraordinary mode propagation the plasma volume is divided into three regions (see Fig. 6). These regions are defined as follows:

(1) Region I consists of surface plasma on the low magnetic field side of the extraordinary mode cutoff. It is bounded by the low field cavity wall $S_{\text {wall }}^{\mathrm{I}}$, by the magnetic field line separating the surface and core plasma $S^{1, I I}$, and by the portion of the cutoff surface contained in the surface plasma $S_{s}$.

(2) Region II consists of core plasma on the low field side of the cutoff. It is bounded by $S^{\mathrm{I}, \mathrm{II}}$ and the portion of the right-hand cutoff contained in the core plasma.

(3) Region III consists of all plasma (surface and core) on the high field side of the cutoff. It is bounded by the cutoff and by the high field cavity wall $S_{\text {wall }}^{\mathrm{III}}$.

For purposes of the power balance model the annulus is considered to lie on a portion of $S^{I, I I}$, the boundary between regions I and II. Another surface that plays an important role in the power balance model is the fundamental cyclotron resonance surface. This surface lies in region III and is divided into two parts, $S_{s}$ and $S_{c}$, which intercept the surface and core plasma components, respectively.

It is assumed that each mode propagates freely inside each region. At a wall boundary surface the waves are reflected and partially converted to the other mode. At a boundary surface in the plasma, waves can be reflected, partially absorbed, and partially transmitted to the adjacent plasma region. The power flux for each mode in each region is assumed to be isotropic in angle and uniform in space. Therefore, the Poynting flux for the $j$ th mode is $S^{j}(x, k)=V_{g} W_{j}$, where $V_{g}$ is the group velocity and $W_{j}$ is the $j$ th mode energy density. For simplicity we assume $V_{g} \sim c$ and in the direction of $k$ so that the $j$ th mode power flux on a surface $S$ is

$$
P_{\text {incident }}=\int_{S} d \sigma \int_{k} d^{3} k S_{j}(x, k)=\frac{c}{4} S W_{j}
$$

Energy conservation is enforced by relating the flux of power into and out of each region, yielding a set of linear equations that determines the four energy densities $W_{x}^{\mathrm{I}}, W_{x}^{\mathrm{II}}, W_{x}^{\mathrm{III}}$, and $W_{o}$ in terms of the power sources. Since a previous version of the model has been presented in Ref. 4, 

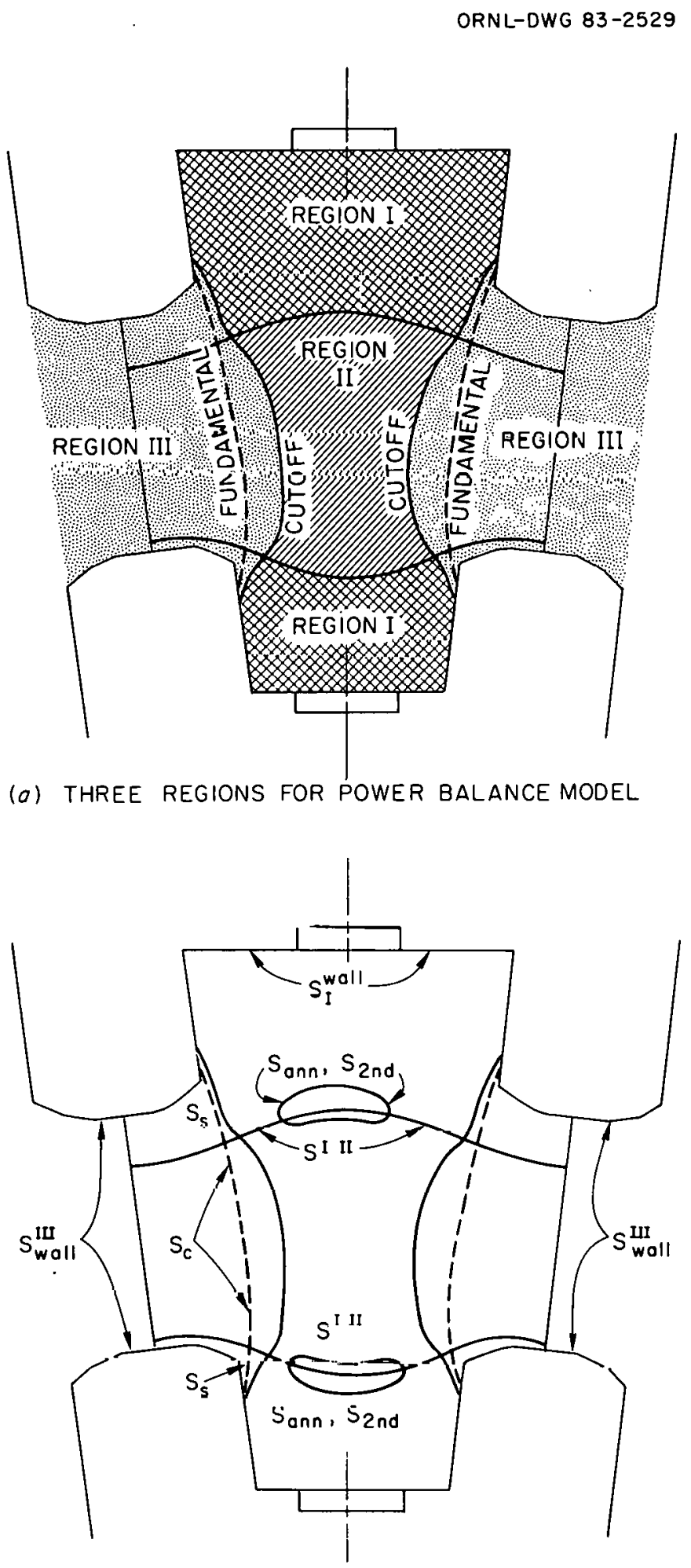

(b) IMPORTANT SURFACES FOR POWER BALANCE MODEL

Fig. 6. Geometry of the three plasma regions and critical surfaces defined for the power balance model. 
the discussion of the power balance equations is relegated to the Appendix [see Eqs. (A.10)-(A.13)]. The power deposited in the three plasma components can be obtained from the energy densities [see (Eq. (A.14)]. The input parameters to the model are the areas of the various boundary surfaces, the averaged fractional absorptions $\langle f\rangle$, the averaged mode conversion coefficients $\left\langle t_{i j}\right\rangle$, and the average Budden tunnelling coefficient $\mid T^{2}$. Methods used to estimate these parameters and sensitivity of the results to the assumptions are discussed in Ref. 4. The values used here are presented in Table II.

Table II. Input parameters for power balance model.

\begin{tabular}{|c|c|c|c|}
\hline & EBT-I & EBT-S & EBT-P \\
\hline$S_{c}\left(\mathrm{~cm}^{2}\right)$ & 795 & 632 & 2128 \\
\hline$S_{c}\left(\mathrm{~cm}^{2}\right)$ & 1010 & 938 & 461 \\
\hline$S^{\mathrm{i}, \mathrm{II}}\left(\mathrm{cm}^{2}\right)$ & 1378 & 1077 & 4005 \\
\hline$S_{\text {wall }}^{\mathrm{l}}\left(\mathrm{cm}^{2}\right)$ & 3300 & 3300 & $1.48 \times 10^{4}$ \\
\hline$S_{\text {wall }}^{\mathrm{III}}\left(\mathrm{cm}^{2}\right)$ & 880 & 880 & $4.59 \times 10^{3}$ \\
\hline$S_{a n n}\left(\mathrm{~cm}^{2}\right)$ & 961 & 751 & $2.27 \times 10^{3}$ \\
\hline$S_{2 n d}\left(\mathrm{~cm}^{2}\right)$ & 505 & 394 & $2.27 \times 10^{3}$ \\
\hline$\left\langle f_{a n n}^{x}\right\rangle$ & 0.162 & 0.118 & 0.041 \\
\hline$\left\langle f_{2 n d}^{x}\right\rangle$ & 0.0135 & 0.015 & 0.83 \\
\hline$\left\langle f_{\text {fund }}^{x}\right\rangle$ & 1.0 & 1.0 & 1.0 \\
\hline$\left\langle f_{a n n}^{o}\right\rangle$ & 0.0176 & 0.01 & 0.011 \\
\hline$\left\langle f_{2 \text { nd }}^{0}\right\rangle$ & 0.0 & 0.0 & 0.03 \\
\hline$\left\langle f_{\text {fund }}^{o}\right\rangle$ & 0.016 & 0.06 & 0.291 \\
\hline$\left\langle t_{o x}\right\rangle^{1},\left\langle t_{o x}\right\rangle^{I I I}$ & 0.5 & 0.5 & 0.5 \\
\hline$F_{\mu}(\mathrm{GHz})$ & 18 & 28 & 60 \\
\hline
\end{tabular}

The average fractional absorptivity for the annulus to each mode is determined by tracing a collection of rays covering all solid angles from a point just outside the annulus. The fraction of power absorbed in one passage through the annulus, $f_{a n n}^{j}(\theta, \phi)$, is calculated using the relativistic damping code, and this fraction is averaged over a solid angle to obtain \langle\rangle$_{a n n}^{j}$. The effective surface area of the annulus $S_{a n n}$ is taken as the area of a cylinder of radius $r_{p}$ and length equal to the half-pressure annulus length $\left(l_{A}=11 \mathrm{~cm}\right.$ for EBT-I/S). A similar model is used to treat core heating at the second harmonic, except that the relevant surface area $S_{2 n d}$ is taken as that part of the second harmonic surface which lies in the core plasma.

Using the parameters shown in Table II we have calculated the deposition of the power remaining after one pass through the plasma. Of this power $100 \%$ of the extraordinary mode remains in region $I$, whereas $68 \%$ of the ordinary mode remains in region I and $30 \%$ is transmitted to region III. The results are summarized in Table III.

Figure 7 shows the fraction of $P_{i n j}$ deposited in each plasma component as a function of $\left\langle n_{\text {surf }}\right\rangle$, the average density of the surface plasma at the fundamental resonance in EBT-S. We have plotted the heating as a function of $\left\langle n_{\text {surf }}\right\rangle$ because the results are somewhat sensitive to this parameter, whose value is not accurately known. The effect of $\left\langle n_{\text {surf }}\right\rangle$ is to contrnl the amount of Budden tunnelling and absorption in the low density surface plasma. As discussed in Refs. 4 and 8, for a wide range of angles of incidence, the Budden tunnelling transmission coefficient in a parallel stratified plasma is given by

$$
\begin{aligned}
& |T|^{2}=\exp \left(-\pi K_{o} X_{o}\right), \\
& K_{o} X_{o}=\frac{\omega}{c} L_{z} \frac{\omega_{p e}^{2}}{\Omega_{e}^{2}},
\end{aligned}
$$


Table III. Final results for power deposition/input power.

\begin{tabular}{llll}
\hline \multicolumn{1}{c}{ EBT-I } & Surface & Core & Rings \\
\hline Single pass & 0 & 0 & 0.05 \\
Randomized & 0.33 & 0.25 & 0.36 \\
Total & 0.33 & 0.25 & 0.41
\end{tabular}

\section{EBT-S}

\begin{tabular}{llll} 
Single pass & 0 & 0.01 & 0.03 \\
Randomized & 0.39 & 0.32 & 0.25 \\
Total & 0.39 & 0.33 & 0.28 \\
\hline
\end{tabular}

Including distribution system effïciency, $\eta \simeq 0.75$ for EBT-S

$$
P_{\text {surf }}=0.30 P_{\text {tube }}, P_{\text {core }}=0.25 P_{\text {tube, }}, P_{\text {ring }}=0.21 P_{\text {tube }}
$$

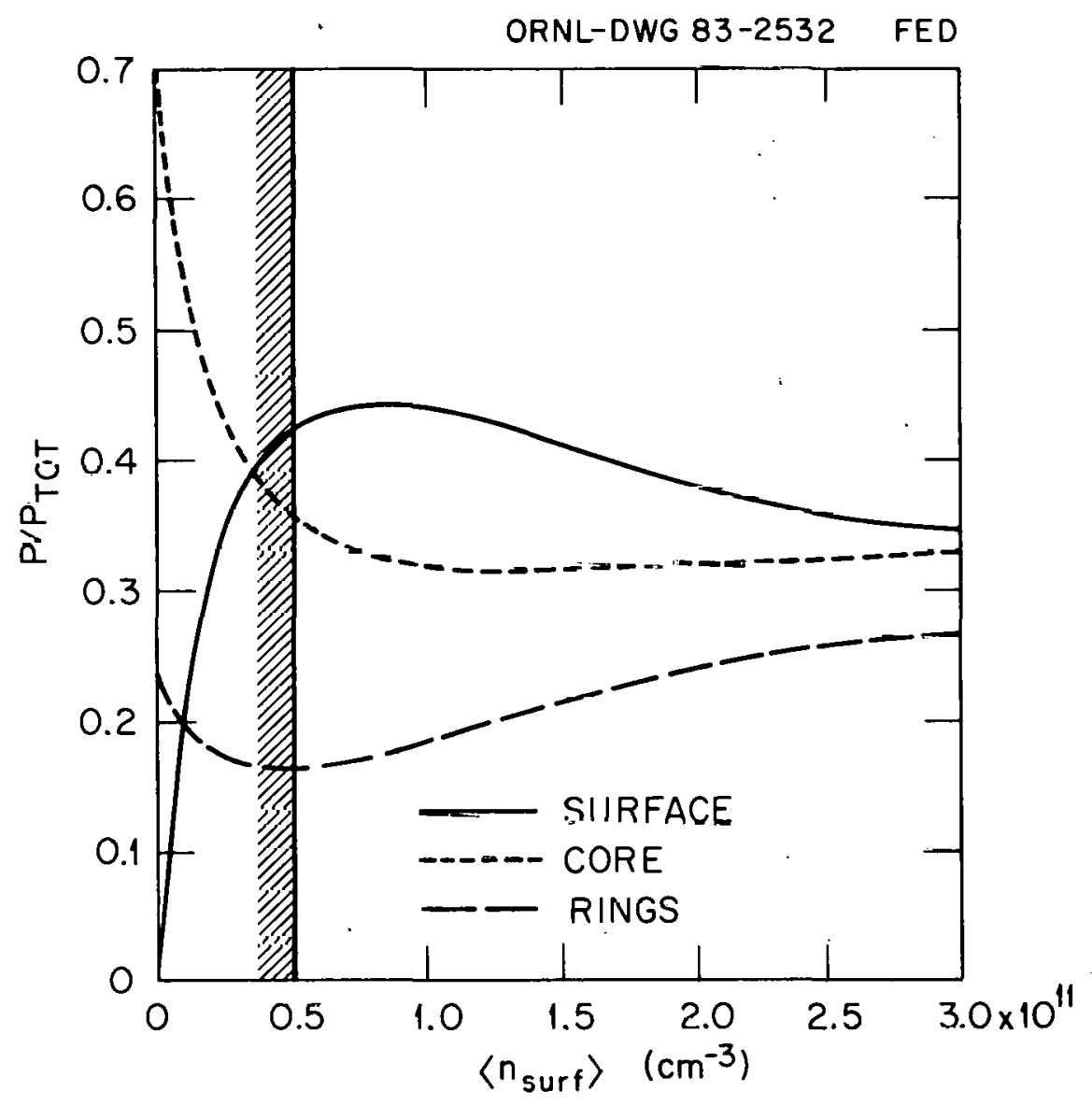

Fig. 7. Power depusited in each plasma component vs average surface plasma density. 
where $L_{z}$ is the magnetic field scale length and $\omega_{p e}$ is the plasma frequency at the resonance. A good estimate for $L_{z}$ in the surface of EBT-I is $-7.5 \mathrm{~cm}$ at resonance. Unfortunately, there do not exist definitive density measurements in the surface plasma of EBT. For low T-mode operation (such as the specific case we are attempting to model here), multichord interferometer measurements show the plasma density down from the peak value by about one-half at the ring location. This would give $n_{\text {surf }}=2-3 \times 10^{11} \mathrm{~cm}^{-3}$ at the inside edge of the surface plasma. Although there are large uncertainties, Langmuir probe measurements suggest that $n_{\text {surf }}$ may be as low as $5 \times 10^{10} \mathrm{~cm}^{-3}$ near the wall at the midplane. It is difficult to extrapolate this to the resonant zone, but $5 \times 10^{10} \mathrm{~cm}^{-3} \leqslant\left\langle n_{\text {surf }}\right\rangle \leqslant 3 \times 10^{11} \mathrm{~cm}^{-3}$ seems to be the extreme credible boundary.

We see from Fig. 7 that the results (particularly $P_{\text {core }}$ ) are relatively insensitive to $\left\langle n_{\text {surf }}\right\rangle$ provided $\left\langle n_{\text {surf }}\right\rangle \geq 5 \times 10^{10} \mathrm{~cm}^{-3}$. In the limit $\left\langle n_{\text {surf }}\right\rangle \rightarrow 0$, the power to the surface converges to zero as it should and is transferred predominantly to the core. At low density the right-hand cutoff in the surface becomes an open window for the extraordinary mode to the core. At higher density, $\left\langle n_{\text {surf }}\right\rangle \geqslant 3 \times 10^{11} \mathrm{~cm}^{-3}$ (or shorter wavelength), the cutoff becomes essentially opaque and Budden tunnelling plays no role in the heating.

In Fig. 8, we show the sensitivity of the results to the assumed value of the average mode conversion coefficient $\left\langle t_{o x}\right\rangle$. As discussed in Ref. 4, studies of the reflection boundary value problem and consideration of other mode randomizing processes lead one to expect that $\left\langle t_{o x}\right\rangle=0.5$. However, Fig. 8 shows that equipartitioning of mode energy is so rapid (due to a large wall surface area) that the results are not sensitive to $\left\langle t_{o x}\right\rangle$, provided $\left\langle t_{o x}\right\rangle \geqslant 0.05$.

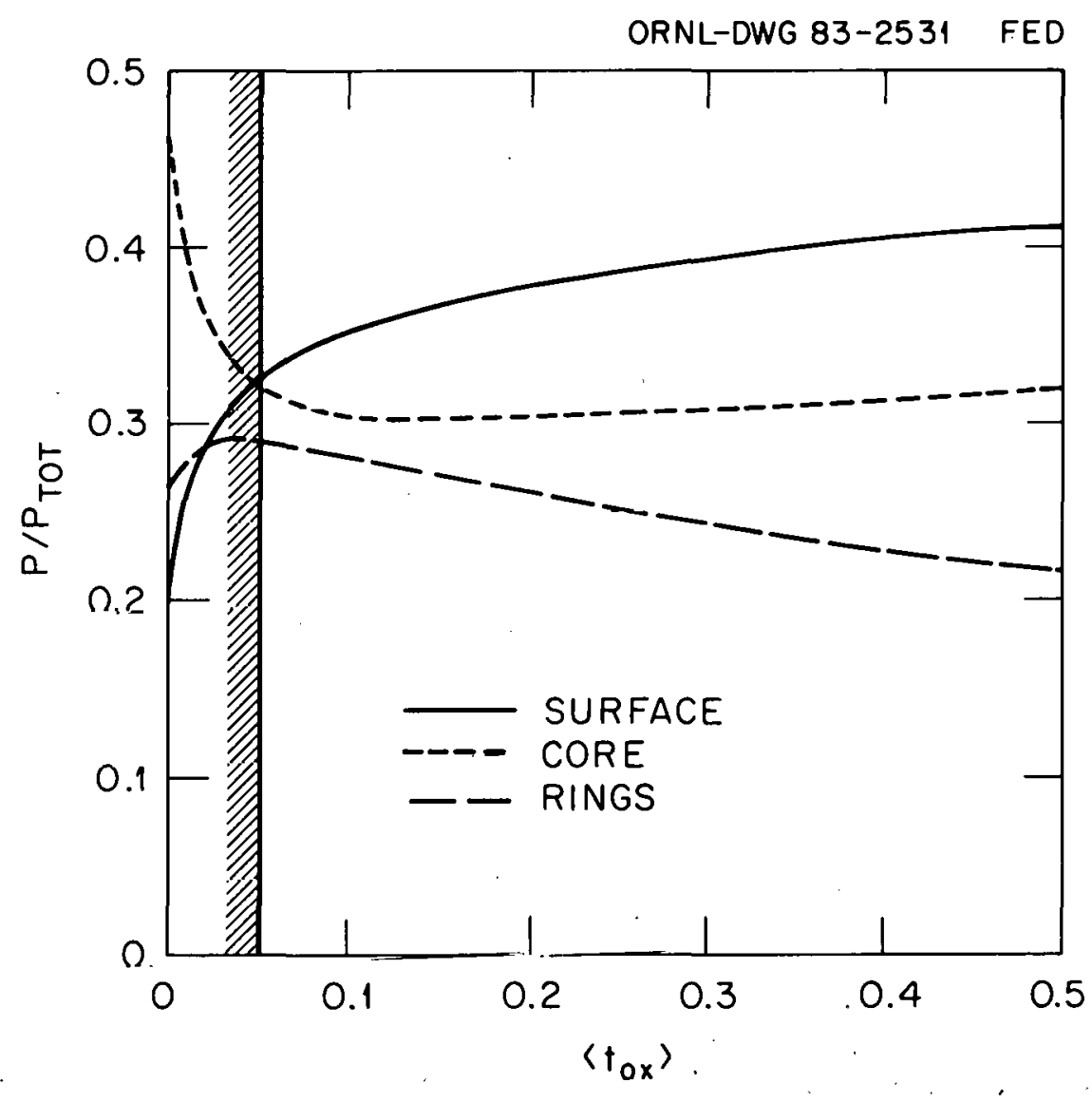

Fig. 8. Power deposited in each plasma component vs average wall reflection mode conversion coefficient. 


\section{EXPERIMENTAL MEASUREMENTS}

The microwave power flux on the cavity wall of EBT-I/S has been measured using a simple water load calorimeter, Fig. 9. A $25-\mathrm{W}$, cw $18-\mathrm{GHz}$ source coupled directly to the calorimeter was used for calibration. These measurements showed that reflections from the calorimeter were less than $1 \%$ and that essentially all of the microwave power incident on the aperture of the calorimeter is absorbed by the circulating water load. The temperature rise of the water is therefore an accurate measure of the microwave intensity at the cavity wall.

ORNL-DWG 82-3280 FED

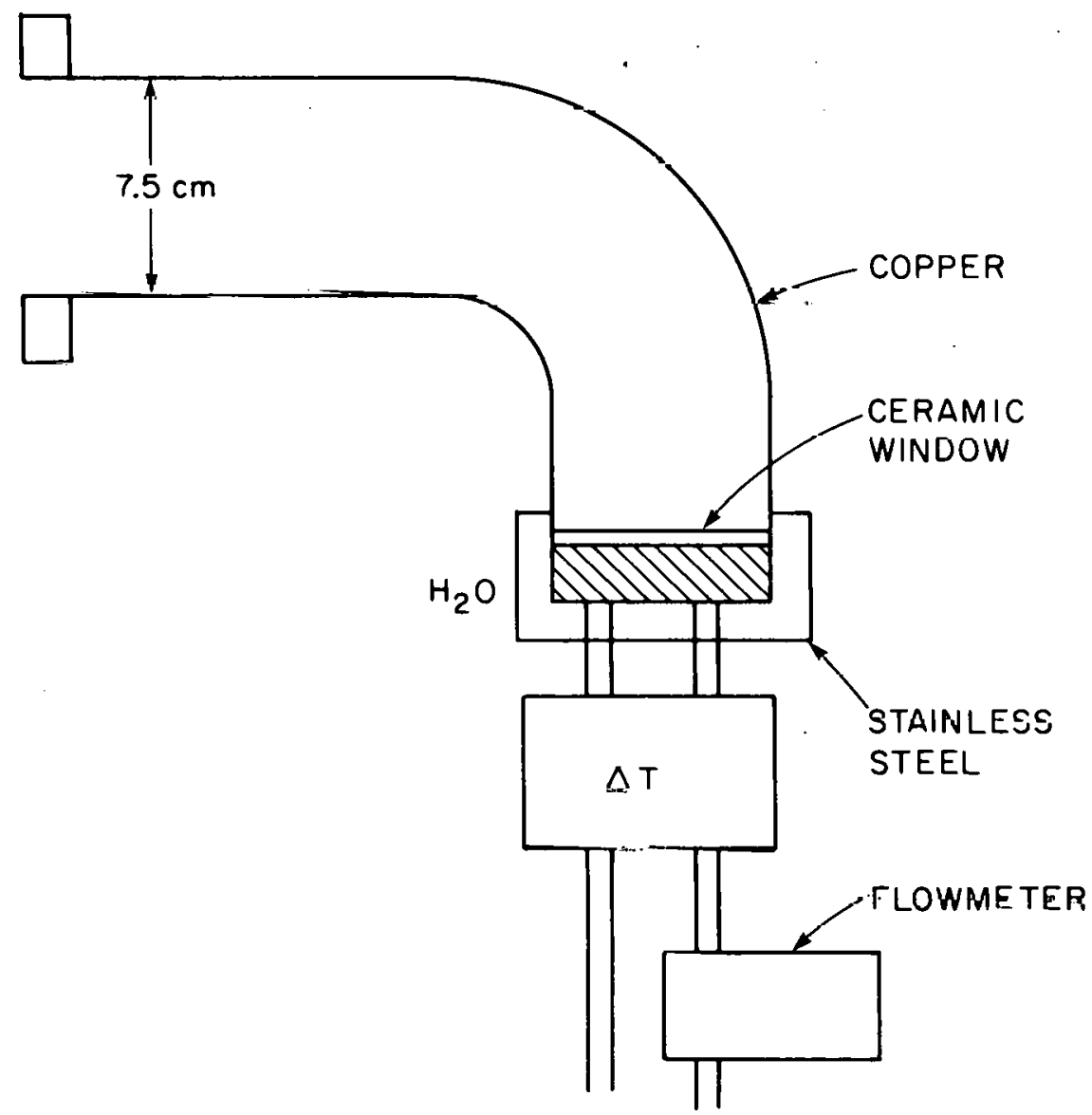

Fig. 9. Schematic diagram of microwave calorimeter.

Figure 10(a) shows the measured culourimctcf power and ring stinred energy $W_{\mathrm{I}}$. as a function of torus gas fill pressure for a typical EBT-S run with $100 \mathrm{~kW}$ of $28-\mathrm{GHz}$ power. As the pressure is reduced, $W_{\perp}$ increases and operation moves from the C-mode $\left(P_{o}>14 \times 10^{-6}\right.$ torr $)$ to the T-mode. At pressures below $4 \times 10^{-6}$ torr as shown, the unstable M-mode appears. (For additional discussion of the various operating modes in EBT, see Ref. 9.) Reducing the gas pressure changes the plasma parameters that affect microwave propagation and absorption and thereby alter the microwave flux at the cavity wall. Specifically, ring density increases, which increases ring absorption; core temperature increases, which increases fundamental absorption by the ordinary mode as well as core absorption at the second harmonic; and the density profile steepens, which may affect Budden tunnelling in the surface plasma. 

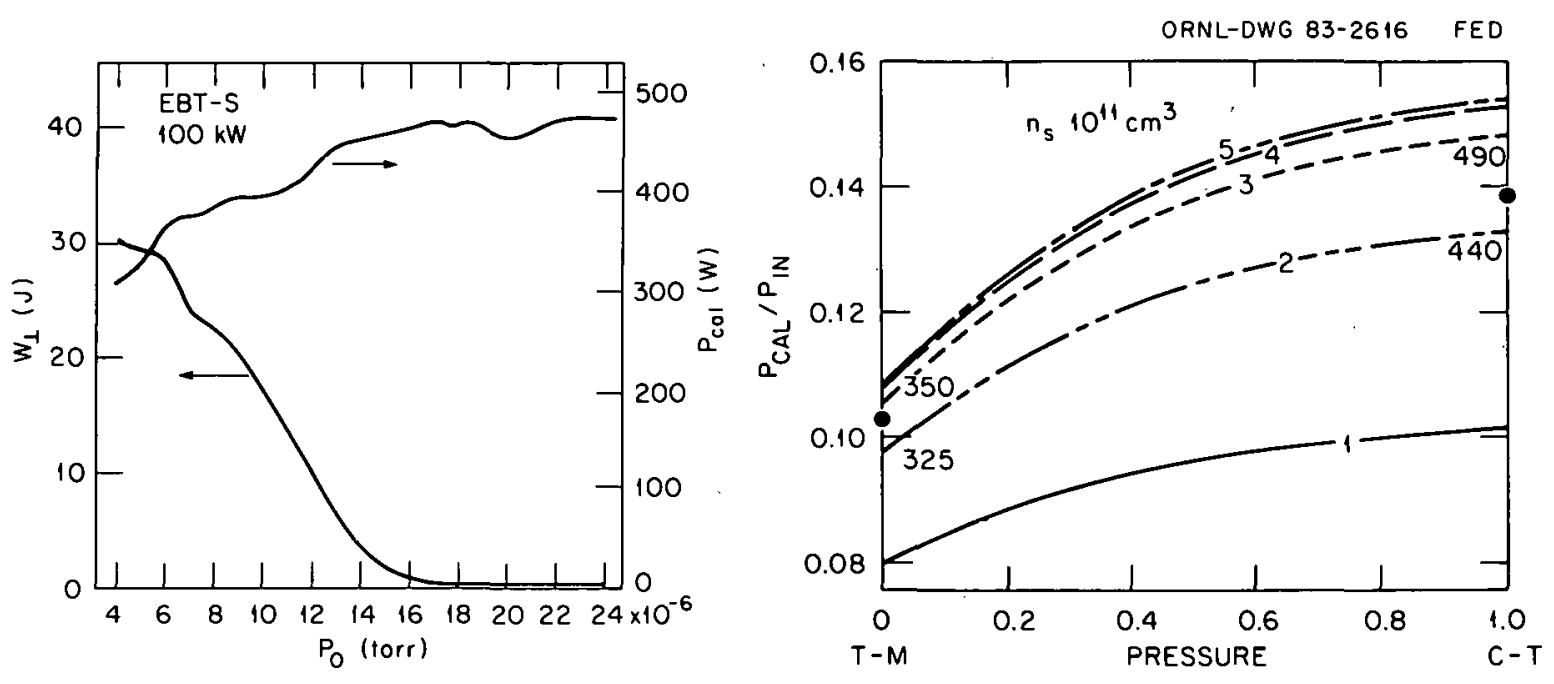

Fig. 10. (a) Measured power into calorimeter $P_{c a l}(\mathrm{~W})$ and ring stored energy (J) vs fill pressure. (b) $P_{\text {cal }} / P_{\text {in }}$ as calculated from power balance model vs artifical pressure variable. The data points indicate measured $P_{c a l} / P_{\text {in }}$ at the T-M transition and $\mathrm{C}-\mathrm{T}$ transition.

To make a comparison with theory, we have included the calorimeter aperture as a loss term in region I of the power balance model. The variation of fill pressure is modeled by introducing a dummy pressure variable $P_{o}$ such that for $P_{o}=0$ the ring and core absorption coefficients have the appropriate T-mode values, as discussed in Sec. V. As $P_{0} \rightarrow 1$, the ring and second harmonic absorption coefficients decrease exponentially to zcro. At the high pressure (C-mode) the only microwave sinks are extraordinary mode absorption in region III at the fundamental and absorption by the calorimeter itself. Figure 10(b) shows the fraction of total injected power absorbed by the calorimeter $P_{c a l} / P_{i n j}$ as a function of $P_{o}$ for various assumed values of average surface plasma density $\left\langle n_{\text {surf }}\right\rangle$. We immediately see the trend from Fig. 10(a) of $P_{\text {cal }}$ decreasing with decreasing $P_{o}$ (i.e., with increasing ring energy). Also shown in Fig. $10(\mathrm{~b})$ are the experimental values of $P_{c a l} / P_{i n j}$ at the pressure extremes. To determine $P_{i n j}$ we have included a distribution system efficiency of $\eta_{\text {dist }}=0.75$. At the T-mode end $\left(P_{o}=0\right)$ the theoretical results for $1.5 \times 10^{11} \mathrm{~cm}^{-3} \leqslant\left\langle\mathrm{n}_{\text {surf }}\right\rangle<\infty$ agree to within the experimental uncertainty. At the high pressure end the theory agrees within experimental uncertainty for $1.5 \times 10^{11} \mathrm{~cm}^{-3} \leqslant\left\langle n_{\text {surf }}\right\rangle \leqslant$ $3 \times 10^{11} \mathrm{~cm}^{-3}$. For a higher surface plasma density $\left\langle n_{\text {surf }}\right\rangle=\infty$, the theory differs from the mean experimental value of $P_{c a l} / P_{i n j} \sim 0.13$ by about 0.02 . The agreement is within about $20 \%$ for $\left\langle n_{\text {surf }}\right\rangle$ as low as $1.0 \times 10^{11} \mathrm{~cm}^{-3}$. We present this curve for comparison although from arguments presented in Sec. V we regard this value to be too low for EBT-S.

In EBT-I operation the $18-\mathrm{GHz}$ microwave system has the flexibility to turn off the power to an individual cavity. Experiments with varying heating configurations were performcd to investigate the flow of power between adjacent cavities. Three cases were considered:

case 1 - Standard operating mode with all cavities fed.

case 2 - Calurimeter cavity off, but all other cavities fed. Only ordinary mode is transmitted past the fundamental resonance into region $I$.

case 3 - Adjacent cavities off, but calorimeter cavity fed.

The results are shown in Fig. 11 as a function of torus fill pressure. Also shown are points indicating $P_{c a l} / P_{i n j}$ predicted by the power balance model. For cases 2 and 3 the nonsymmetric flow of power between fed and unfed cavities is self-consistently included in the calculation. The top curve (all fed) is the EBT-I equivalent of Fig. 10(a) for EBT-S. The agreement with experiment is excellent at the low pressure point for all three experimental cases. There is much less dependence 
of $P_{c a l} / P_{i n j}$ on $P_{o}$ in EBT-I than in EBT-S. Correspondingly, the theory overestimates the calorimeter power at the high pressure end. Nevertheless, we regard the agreement between theory and experiment as quite good. These results tend to confirm the model assumptions related to wave trapping by the right-hand cutoff, strong absorption of extraordinary mode at the fundamental resonance, and conversion between ordinary and extraordinary mode, since these processes dominate the transmission of power between cavities.

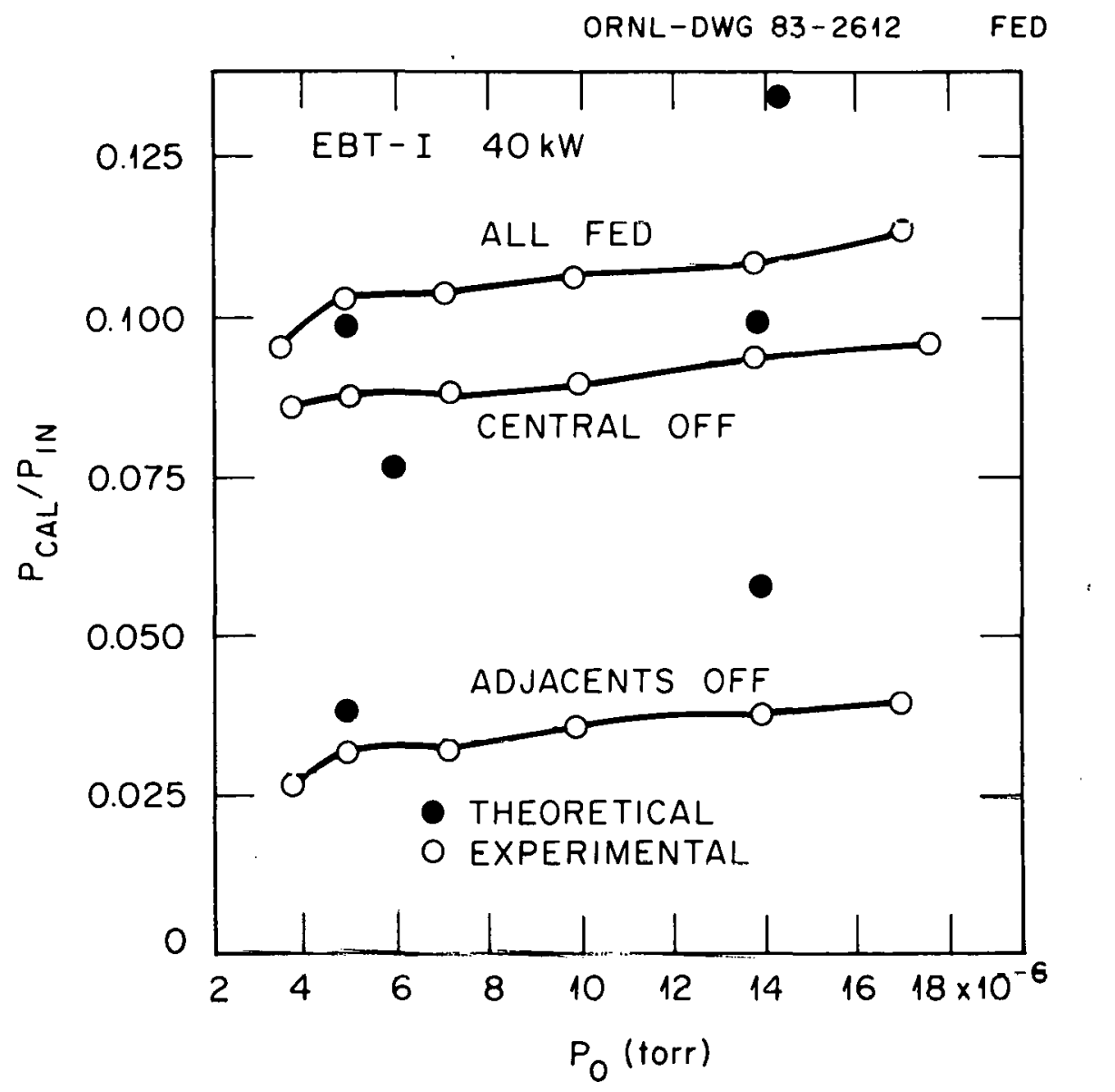

Fig. 11. Measured power into the calorimeter vs pressure for various cavity feed configurations. Dots indicate $\boldsymbol{P}_{c a l} / \boldsymbol{P}_{\text {in }}$ calculated from power balance model at C-T and T-M transitions for the different feed configuratiuns.

$\Lambda$ sel of mcnsurements wạs also made with a fundamental mode waveguide stub ieceiver placed at the edge of the cavity. The stub was mounted in a vacuum ball jolnt that allowed it to be swept $5 \mathrm{~cm}$ across the cavity port opcning. This stub has a broad antenna pattern, which does not vary significantly over the $\pm 9^{\circ}$ sweep angle. Figure 12 shows the result when the cavity is unfed and only the power coupled through the throats is measured. The lack of spatial structure seen in Fig. 12 supports the assumption of the pnwer balance model that multiple wall reflections result in an isotropic power density. Rotating the waveguide stub through $90^{\circ}$ in the feedthrougli allowed measurements of both the 0 -mode and $\mathrm{X}$-mode polarizations. For the unfed configuration the measured power is essentially independent of polarization. Because very little of the X-mode polarization can propagate through the mirror throats (X-mode is nearly $100 \%$ absorbed at the fundamental resonance), mode conversion upon reflection must indeed be a strong effect. 


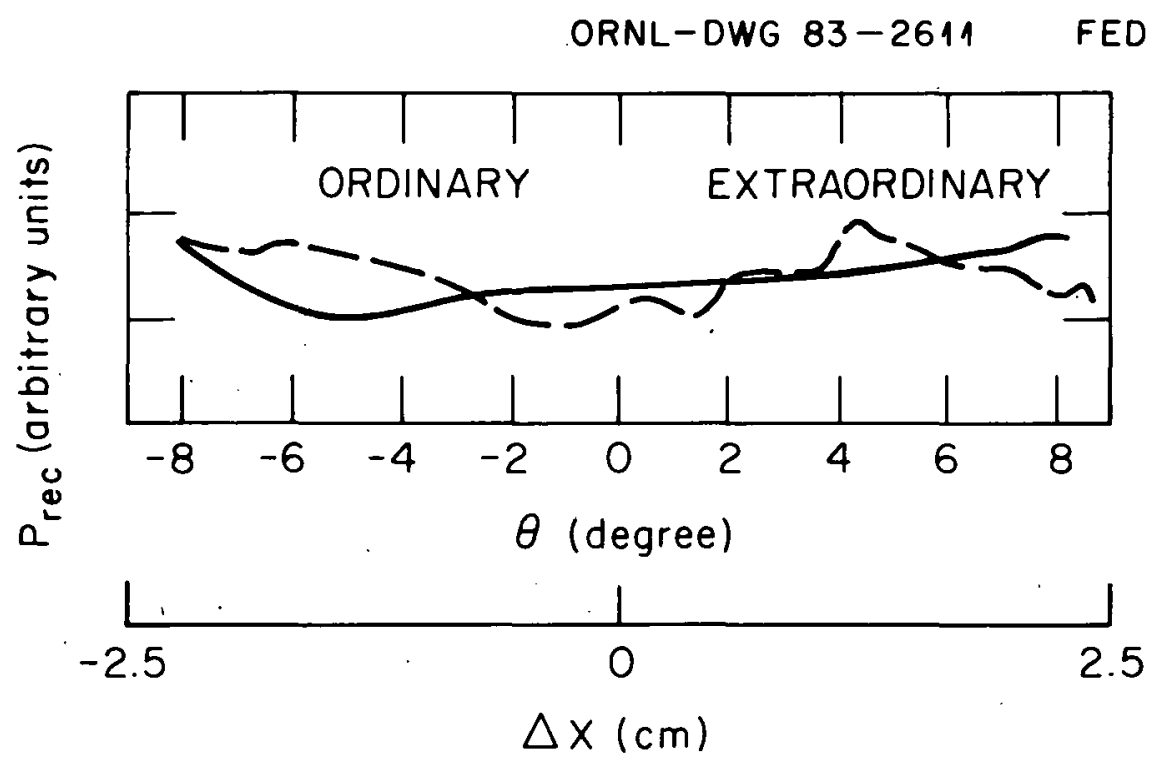

Fig. 12. Spatial variations of ordinary and extraordinary mode power received by a waveguide stub. Cavity was unfed.

The ability of the power balance model to reproduce the absolute calorimeter power measurements can be considered evidence that the overall rate of power dissipation predicted by the model is approximately correct. That is, there are no important power sinks that are neglected, nor are power sinks included in the model gross overestimates. This does not prove that the partitioning of power between ring, core, and surface plasma is correct. However, the demonstration of correct dependence on $P_{o}$ (the primary factor being ring absorptivity) and correct power flow between cavities certainly lends support to the correctness of the model in detail. It should be emphasized that coupling $-10 \%$ of the injected power into the calorimeter is by no means a trivial result to be expected independent of any plasma wave propagation effects. The surface area of the calorimeter aperture $\left(S_{c a l}-44 \mathrm{~cm}^{2}\right)$ amounts to about $1 \%$ of the wall area in one cavity. If the microwaves were heavily damped such that only one or two wall reflections occurred, then one would expect $P_{\text {call }} P_{\text {inj }}<S_{\text {cal }} / S_{\text {wall }}=0.01$ and much less than this when the calorimeter cavity is unfed. On the other hand, if wave ahsorption by the plasma is very weak then the calorimeter becomes the only sink and one would have $P_{\text {cal }} P_{\text {inj }} \rightarrow 1.0$.

\section{PROJECTIONS FOR EBT-P}

Current plans call for the individual sectors of EBT-P to be gcometrically similar to EBT-I although larger by nearly a factor of 2 , with the mirror ratio again $-2: 1$. To perform ray-tracing calculations for EBT-P we have simply scaled the 2-D equilibrium geometry for EBT-I upward by a factor of 1.9. Again, the peak annulus pressure is placed at the vacuum second harmonic, in this case $r_{\text {peak }}=18 \mathrm{~cm}$. The assumed plasma parameters used in these calculations are: ${ }^{10}$ $n_{c}=1.1 \times 10^{13} \mathrm{~cm}^{-3}, T_{c}=2 \mathrm{keV}, T_{A}=1 \mathrm{MeV}$, and $n_{A}=7 \times 10^{11} \mathrm{~cm}^{-3}$. This gives local beta of $\beta=0.16$. Figure 13 shows midplane profiles of core density, annulus density, and magnetic field for the EBT-P bumpy cylinder equilibrium.

Note the small ratio of $\left(\omega_{p e}^{2}\right)_{a n n}$ to $\left(\omega_{p e}^{2}\right)_{c o r e}$ and the relatively large separation bctween the vacuum and finite-beta second harmonic resonance. This places the finite-beta second harmonic layer near the maximum of core density and temperature. Figure 14 shows a cross section of the EBT-P bumpy cylinder model. EBT-P was designed to make more efficient use of the microwavc power by minimizing the area of the fundamental resonance in the surface plasma. 


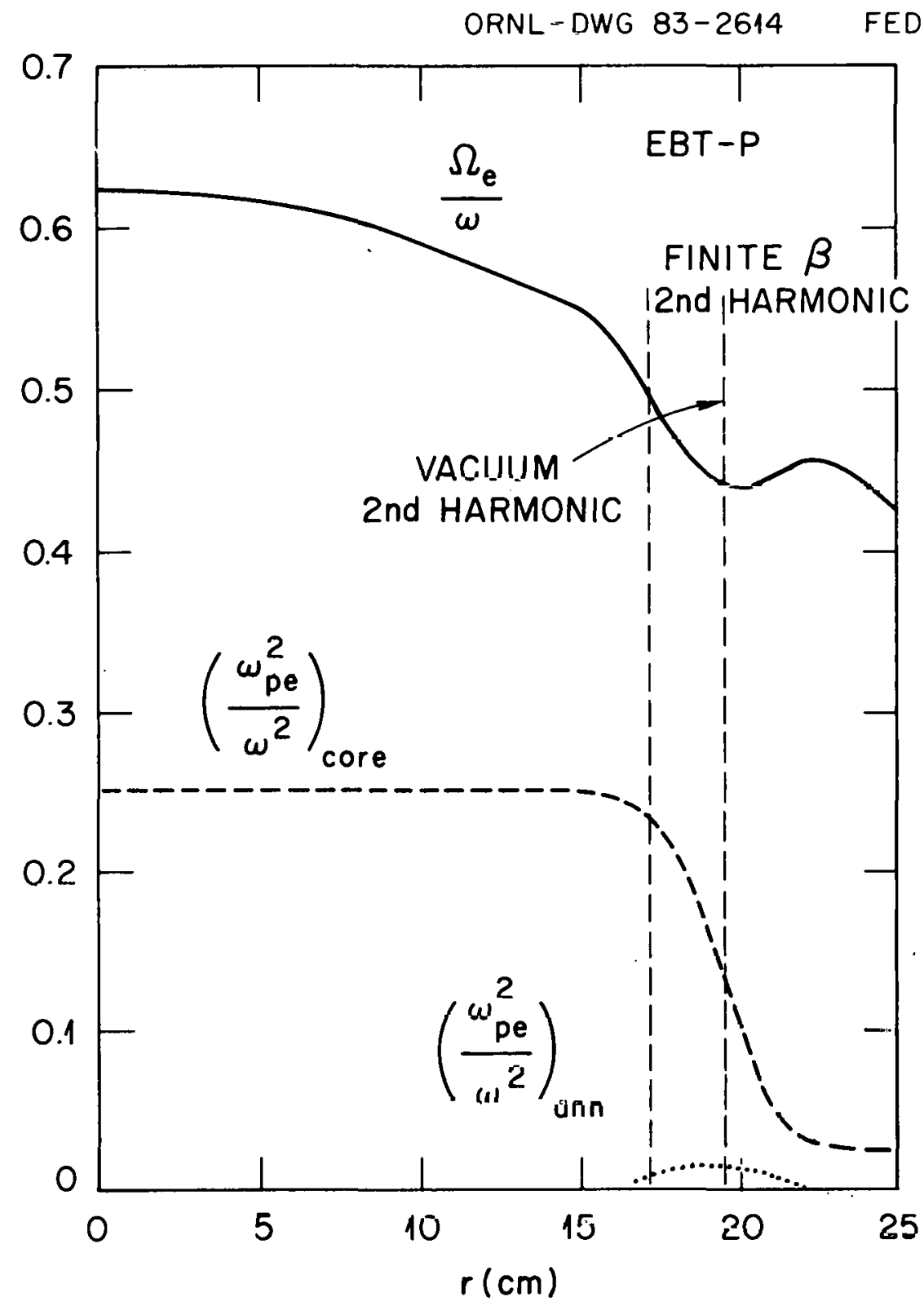

Fig. 13. Midplane profiles of core density, annulus density, and magnetic field for the EBT-P bumpy cylinder equilibrium used in ray tracing.

Figures 15(a) and 15(b) show typical ray paths for the extraordinary mode in the equatorial plane and midplane respectively. Again, $f_{a n n}^{x}$ and $f_{2 n d}^{x}$ are shown in parentheses by each ray. One sees a dramatic difference relative to the EBT-I calculations between annulus absorption and second harmonic core absorption. Even though the ring density is a factor of 3.5 larger and the ring is a factor of 2 thicker than in EBT-I, the fractional ring absurplion in EBT-P is nearly an order of magnitude smaller. This is due entirely to the rapid decrease in $k_{i}$ with temperature above $100 \mathrm{keV}$ (see Fig. 3). Also, there is tremendous absorption by the core plasma in a thin layer about the finite-beta second harmonic resonance owning to the high core density and temperature. We see that for rays tangential to the finite-beta second harmonic layer $f_{2 n d}^{x}$ approaches $100 \%$. 


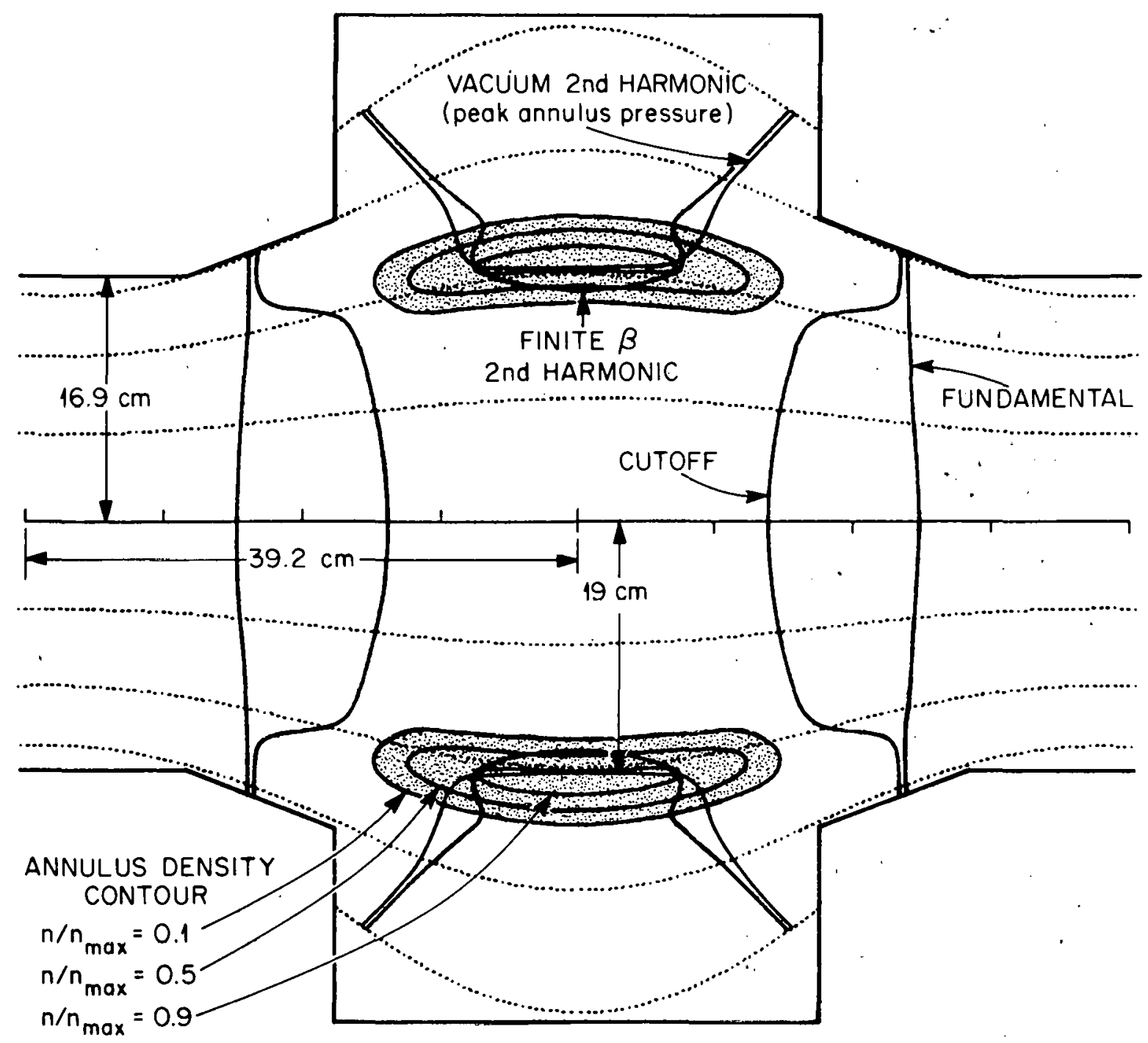

Fig. 14. Cross section of the bumpy cylinder equilibrium for EBT-P usod in ray tracing.

Again, assuming the antenna radiation pattern given in Eq. (9), we find solid-angle-averaged fractional absorption to be

$$
\langle f\rangle_{a n n}^{x}=0.017,\langle f\rangle_{2 n d}^{x}=0.264 \quad \text { (extraordinary mode) }
$$

Figure 16 shows ordinary mode rays in the equatorial plane and midplane. The numbers in parentheses are $f_{a n n}^{o}$ and $f_{2 n d}^{o}+f_{1 s t}^{o}$, respectively. Ordinary mode absorption by the annuli and second harmonic is virtually ignorable although some ordinary mode rays have significant damping at the fundamental resonance. When averaged over the radiation pattern above this gives

$$
\langle f\rangle_{a n n}^{o}=5 \times 10^{-3},\langle f\rangle_{2 n d}^{o}=0,\langle f\rangle_{i s t}^{o}=0.043 \quad \text { (ordinary mode) } .
$$


ORNL-OWG 89-23413A FED

(a) equatorial pLane

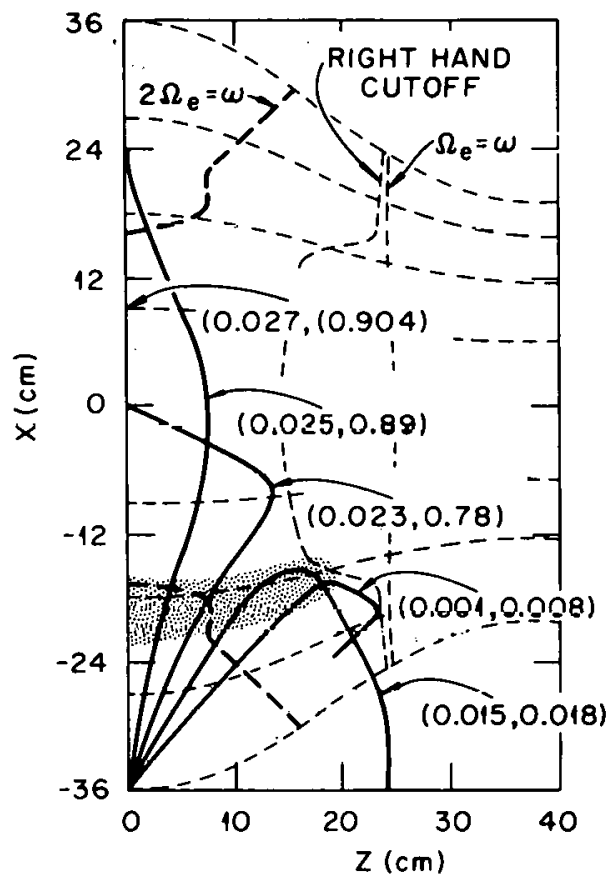

(b) MIDPLANE

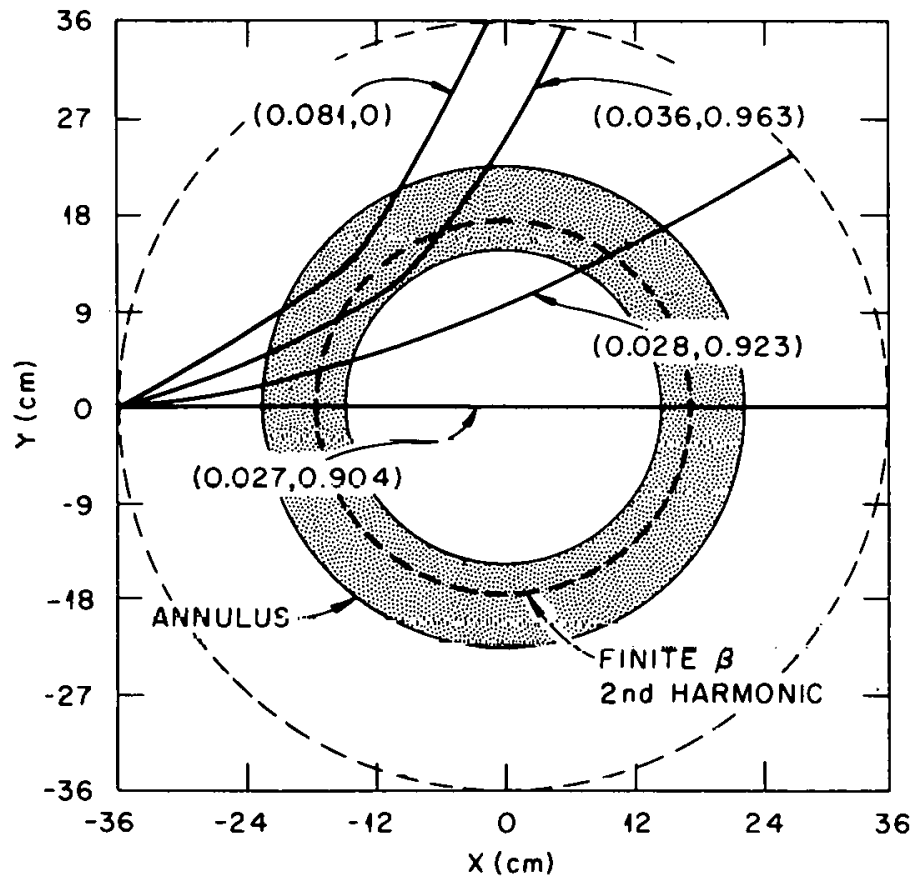

Fig. 15. Ray paths for the extraordinary mode in EBT-P. Numbers in the parentheses are fractions of power absorbed from the ray by the annulus component and by the core component at the second harmonic resonance, respectively.

(a) EQUATORIAL PLANE

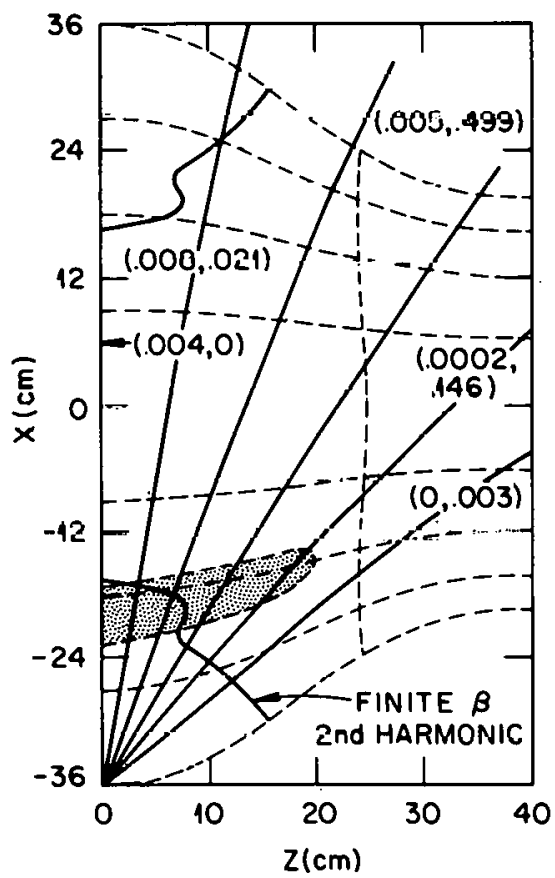

QRNL. - NWG R?-2361 FED

(b) MIDPLANE

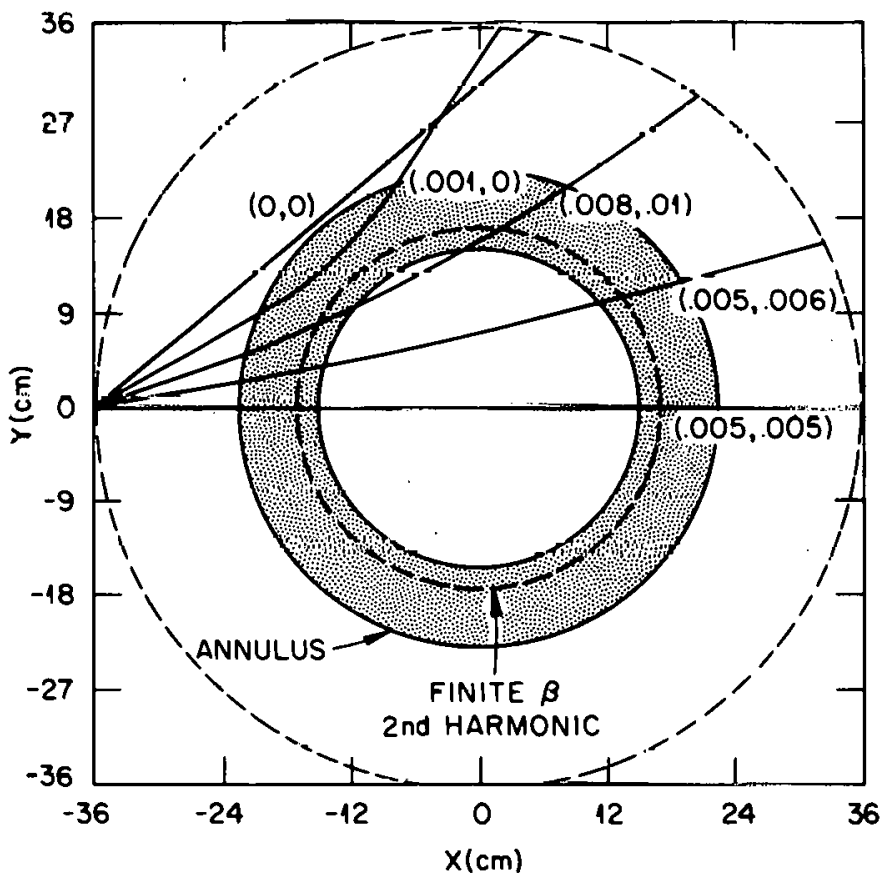

Fig. 16. Ray paths for the ordinary mode in EBT-P. Numbers in the parentheses are fractions of power absorbed from the ray by the annulus component and by the core component (both fundamental and second harmonic), respectively. 
Using these results and assuming a 50/50 ordinary/extraordinary mode ratio from the antenna gives for the single-pass absorption

$$
\begin{aligned}
& P_{a n n}=\langle f\rangle_{a n n}^{x} P_{i n j}^{x}+\langle f\rangle_{a n n}^{o} P_{i n j}^{o}=9 \times 10^{-3} P_{i n j}, \\
& P_{2 n d}=\langle f\rangle_{2 n d}^{x} P_{i n j}^{x}=0.132 P_{i n j}, \\
& P_{1 s t}=\langle f\rangle_{1 s t}^{o} P_{i n j}^{o}=0.022 P_{i n j} .
\end{aligned}
$$

Of the $83.2 \%$ not absorbed in one pass, all of the extraordinary mode or $0.359 P_{i n j}$ remains in region I while $32 \%$ of the ordinary mode or $0.15 P_{i n j}$ appears in region III.

Again, we have calculated the deposition of the remaining power using the 0-D power halance model. The input parameters to the model are presented in Table II. There are important changes compared to the parameters for EBT-I/S; specifically, the average fractional absorptions by the annuli are reduced, the average fractional absorptions by the core at second harmonic and fundamental are increased, and the ratio $S_{s} / S_{c}$ is greatly reduced. With these parameters we find for single-pass and multipass power deposition

$$
\begin{array}{ll}
P_{\text {core }}^{f \text { und }}=0.38 P_{i n j} & P_{\text {core }}^{2 n d}=0.54 P_{i n j} \\
P_{\text {surf }}=0.04 P_{i n j} & P_{\text {ann }}=0.04 P_{i n j} .
\end{array}
$$

The large loss of power in the surface plasma at the fundamental seen in EBT-I/S is now greatly reduced. However, there is strong absorption by the core component at the second harmonic resonance. What amount of this power is conducted into the plasma center as opposed to being lost to the surface is highly dependent on the profile of the electron thermal conductivity. In turn, the thermal conductivity is quite sensitive to the temperature, density, ambipolar potential, and magnetic field profiles. It should be emphasized that the second harmonic absorption itself is very sensitive to details of the profiles. For example, by assuming that the annulus peak is at the vacuum second harmonic, we have forced the finite-beta second harmonic to occur at high values of core density and temperature (see Fig. 3). However, if the annulus peak were at the finite-beta second harmonic, $k_{i}^{2 n d}$ would be reduced by about a factor of 4 . On the other hand, the flatness of the magnetic field profile at the annulus peak would tend to increase the integrated absorption. Another complicating factor is that isotropy of the microwaves may not be a good assumption due to strong absorption by the second harmonic for some directions of propagation. This has partially been accounted for by the inclusion of region II in the model.

\section{CONCLUSIONS}

We have presented ray-tracing calculations which show that in EBT-I/S as well as in the projected EBT-P only a small fraction of the injected microwaves are absorbed in a single pass through the plasma. The remaining power, which makes many wall reflections and many transits through the plasma, has been described by a simple 0-D wave power balance model. This model shows that in EBT-I and EBT-S a relatively small fraction (20\%-30\%) of the total injected microwave power is absorbed by the core plasma, whereas a larger fraction is absorbed by the poorly confined surface plasma. Theoretical calculations of wave power incident on the cavity walls are in good agreement with calorimeter measurements for a variety of operating conditions. This indicates that the overall rate of power dissipation predicted by the theory is correct and gives us confidence that the calculated partitioning of power between surface, core, and annulus plasmas is meaningful. 
An area of possible discrepancy lies in the powcr absorbed by the rings. The rings in EBT tend to have quite long lifetimes after power turnoff or turndown with characteristic decay time $\tau \sim 100-200 \mathrm{~ms}$ in EBT-I. ${ }^{11}$ If one simply assumes $P_{a n n}=W_{\perp} / \tau$, then for the 8-J ring modeled above the power loss (and perforce the power absorbed) would be $P_{\text {ann }} \sim 40-80 \mathrm{~W}$. For comparison, when the classical losses (drag, scattering, and synchrotron radiation) are integrated over the assumed ring and core plasma profiles modeled in Sec. II, one obtains $P_{\text {classical }} \sim 40 \mathrm{~W} / \mathrm{ring}$. Now, with $40 \mathrm{~kW}$ of klystron power and a microwave distribution system efficiency of $66 \%$, the power injected into each cavity is $P_{i n j}-1.1 \mathrm{~kW}$. Then, using $P_{a n n}=0.41 \mathrm{P}_{i n j}$ calculated above, we have $P_{a n n}-450 \mathrm{~W}$, a factor of 6-10 above that inferred from the decay time.

We have performed some approximate studies to determine the effect of moving the annulus to the finite-beta second harmonic resonance or allowing for an anisotropic annulus distribution function. These, however, tend to increase rather than decrease the theoretical ring power absorption. If the ring power absorption is actually significantly less than that predicted here, some mechanism must exist whereby the wave damping is vastly reduced from that predicted by Eq. (6). Possibilities for this include finite $\rho / \mathrm{L}$ effects in the linear heating process or nonlinear particle orbit effects such as superadiabaticity. It is conceivable that superadiabatic limits on the heating at high particle energy are the source of the observed $\rho / \mathrm{L}$ temperature scaling in hot electron devices. ${ }^{12}$ However, it is also quite likely that no discrepancy actually exists. The largest contributions to stored energy and synchrotron radiation come from very energetic particles having long lifetimes. On the other hand, the heating and collisional losses involve predominantly lower energy particles. There may be a large flux of power through the system which is not indicated by the ring decay measurements. Because of the definite correlation between significant wave absorption and ring stored energy (Fig. 10) we tend to this latter view.

There are certain effects not included in the model which may be important. Ring anisotropy, profile effects at the second harmonic resonance, and nongeometrical optics heating effects (i.e., finite $\rho / L$ ) have been previously mentioned. Other processes include:

(1) Nonuniform illumination of fundamental ıesonance Since the extranrdinary mode is produced in the high field side at the cavity wall and is strongly absorbed at the fundamental, the wave power density may be peaked at the plasma edge.

(2) Resistive or anomalous absorption in the surface plasma.

(3) Intermediate energy ring components - We have treated the electron distribution as consisting of two Maxwellian components. However, one might expect a more gradual increase in anisotropy and effective temperature with particle energy. Such intermediate components (in the 1- to 20-keV range) might extend well uulside the ring location and he quite dissipative.

Although we have no quantitative models for these processes, we can say qualitatively that each would tond to enhans. ring or surface plasma absorption and decrease power coupled to the core. We therefore regard the estimates of $\boldsymbol{P}_{\text {core }}$ in EBT-I/IS as upper limits on actual curc heating, Core heating in F.BT-I/S by launching unpolarized waves from the cavity midplane is obviously a very inefficient process. Recent calculations have shown that there is a great deal to be gainen hy launching pure extraordinary mode power from the mirror thuat reginn. An experimental program is now under way to do this.

Our calculations for the projected EBT-P show the extreme sensitivity to the profiles and electron distribution function in the vicinity of the second harmonic resonance. Ring heating is much reduced by relativistic effects and by the presence of a strong energy sink at the second harmonic. If we assume a gyrotron power of $1 \mathrm{MW}$ and a distribution system cfficiency of $66 \%$, the calculated value $P_{a n n}=0.04 P_{i n j}$ gives $P_{a n n}=830 \mathrm{~W} /$ ring. By comparison, the classical losses for the equilibria used are $P_{\text {classical }} \sim 8 \mathrm{~kW} /$ ring. This does not imply that rings cannot be formed in an EBT-P size device but that the rings must form at a temperature less than $1 \mathrm{MeV}$ if the traditional unpolarized, undirected midplane microwave launch is used. Note that ring 
absorptivity rises rapidly as $T_{A}$ decreases from $1 \mathrm{MeV}$ whereas $\boldsymbol{P}_{\text {classical }}$ is nearly flat in this temperature range. These results do, however, point out the importance of studying the ring distribution function and investigating heating techniques such as polarization control, directivity, and alternate frequencies to maximize ring heating without driving the core or surface components.

\section{Appendix A. EQUATIONS FOR THE POWER BALANCE MODEL}

With the assumptions outlined in Sec. $\mathrm{V}$, the $j$ th mode power incident on a given surface is given by Eq. (11). Then, the $j$ th mode power absorbed at a resonant surface $S_{R}$ is

$A_{j}=\int_{S_{R}} d \sigma \cdot \underbrace{}_{\underline{k}} d^{3} k f_{j}(\underline{x}, \underline{k}) \underbrace{j}(\underline{x}, k)=\frac{c}{4} S_{R} W_{j}\langle f\rangle_{j}^{S_{R}}$

where

$f_{j}(x, k)=$ fraction power absorbed in $j$ th mode ray passing through resonance,

$\langle\bar{f}\rangle_{j} \bar{S}_{k}=$ solid angle and surface average of $f_{j}(x, k)$.

Similarly, the $i$ th mode power converted to $j$ th mode on reflection from wall surface $S_{w a l l}$ can be written

$$
T_{i j}=\int_{S_{\text {watl }} d \sigma} \cdot \underline{\int}_{\underline{k}} d^{3} k t_{i j}(\underline{x}, \underline{k}) S^{i}(\underline{x}, \underline{k})=\frac{c}{4} S_{w a l l} W_{i}\left\langle t_{i j}\right\rangle^{S_{\text {wat }}},
$$

where

$$
\begin{aligned}
& t_{i j}(x, k)=\text { local mode conversion coefficient, } \\
& \left\langle t_{i j}\right\rangle^{S_{\text {vell }}}=\text { solid angle and surfacc average of } t_{i j}(x, k) .
\end{aligned}
$$

It will be noticed that the separation between the cutoff and the fundamental cyclotron resonance is quite small in the surface plasma. Particularly in EBT-I, the separation can be considerably less than a free space wavelength $(\lambda-1.7 \mathrm{~cm})$. It is then necessary to consider the possibility that the extranrdinary mode energy Budden tumiels through the cutoff and therefore is partially absorbed at the resonance and partially transmitted to the high field region. The extraordinary mode power in region I absorbed by tunnelling to the fundamental resonance can be written

$\int_{S_{s}} d \underline{\sigma} \cdot{\underline{\int_{k}}} d^{3} k|A|^{2} \underline{S}^{x}(\underline{x}, \underline{\underline{k}})=\frac{c}{4} S_{s}\left\langle|A|^{2}\right\rangle W_{x}^{\mathrm{I}}$

and the power transmitted through to region III is

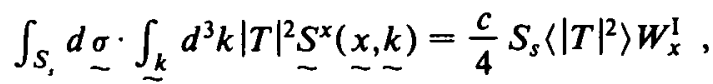

where $|A|^{2}$ and $|T|^{2}$ are the Budden absorption and transmission coefficients, respectively. They are related by $|A|^{2}=|T|^{2}\left(1-|T|^{2}\right)$, The remaining extraordinary modc powei incident on the cutoff is reflected back into region $I$. 
When this process is significant, the power incident on the fundamental resonance from the high field side is not completely absorbed, but a fraction $|T|^{2}$ turnels through the resonance cutoff pair to the low field region. The total power tunnelling to region $I$ is

$$
\int_{S_{r}} d \underline{\sigma} \cdot \int_{\underline{k}} d^{3} k|T|^{2} \underline{S}^{x}(\underline{x}, \underline{\underline{k}})=\frac{c}{4} S_{s}\left\langle|T|^{2}\right\rangle W_{x}^{\mathrm{III}}
$$

There is no reflection for waves incident from the high field side so the power absorbed from region III by the surface plasma is

$$
\int_{S_{s}} d \sigma \cdot \underbrace{}_{\underline{k}} d^{3} k\left(1-|T|^{2}\right) \underline{S}^{x}\left(\underline{x, k)}=\frac{c}{4} S_{s}\left(1-|T|^{2}\right) W_{x}^{\mathrm{III}}\right.
$$

Energy conservation for the extraordinary mode in region I can be expressed as

$$
P_{x}^{\mathrm{I}}+\Gamma_{x}^{S_{1}}+\Gamma_{x}^{S^{1.11}}+T_{o x}^{\mathrm{I}}-T_{x o}^{\mathrm{I}}=0
$$

where $P_{x}^{I}$ is the extraordinary mode power injected into region I (in this case the extraordinary mode remaining in region I after one pass), $\Gamma_{x}$ is the net extraordinary mode power flowing into region I through $S_{s}, \Gamma_{x}^{S^{1} \cdot 1}$ is the net extraordinary mode power entering through $S^{\mathrm{I}, \mathrm{II}}$, and $T_{o x}^{\mathrm{I}}, T_{x o}^{\mathrm{I}}$ are the powers converted from one mode to the other at the wall in region I. Now $\Gamma_{x}^{S_{x}}$ consists of the extraordinary mode power in region III tunnelling through the evanescent region into region I, less the extraordinary mode power in region I tunnelling to region III or absorbed at the fundamental. (The balance of the power incident from region $I$ is reflected at the cutoff.) Using Eqs. (A.3)-(A.5) we obtain

$$
\Gamma_{x}^{S_{s}}=\frac{c}{4} S_{s} W_{i}^{I I I}\left\langle|T|^{2}\right\rangle-\frac{c}{4} S_{s} W_{x}^{1}\left\langle|T|^{2}+|A|^{2}\right\rangle
$$

Similarly, $\Gamma_{x}^{S ! \cdot \prime}$ consists of the extraordinary mode power incident on $S^{\mathrm{l}, \mathrm{II}}$ from region II less the power absorbed by the ring and core at the second harmonic and less the power flowing out of region I into region II,

$$
\bar{\Gamma}^{S^{-1, \prime}}=\frac{c}{4}\left[S^{\mathrm{I} ; \mathrm{II}} W_{x}^{\mathrm{LI}}-\left(S_{a n n}\left\langle\int\right\rangle_{a n n}^{x}+S_{2 n d}\langle f\rangle_{x}^{2 n d}\right) W_{\lambda}^{\mathrm{II}}-S^{\mathrm{I}, \mathrm{II}} W_{x}^{I}\right]
$$

Using these results and Eq. (A.2) we obtain the energy conservation equation

$$
\begin{aligned}
& -\frac{c}{4}\left[S _ { s } \left\langle|T|^{2}+|A|^{2}+S^{\mathrm{I}, \mathrm{II}}+S_{\text {wall }}^{\mathrm{I}}\left\langle t_{x o}\right\rangle^{\left.S_{w a l l}^{\mathrm{I}}\right]} W_{x}^{\mathrm{I}}\right.\right. \\
& +\frac{c}{4}\left[\dot{S}^{\mathrm{I}, \mathrm{II}}-S_{a n n}\langle f\rangle_{a n n}^{x}-S_{2 n d}\left\langle f_{x}\right\rangle^{2 n d}\right] W_{x}^{\mathrm{II}}+\frac{c}{4} S_{s}\left\langle|T|^{2}\right\rangle W_{x}^{\mathrm{III}} \\
& +\frac{c}{4} S_{\text {wall }}^{I}\left\langle t_{o x}\right\rangle^{S_{w a l l}^{\prime}} W_{. o}=-P_{x}^{I}
\end{aligned}
$$




\section{$27 / 28$}

Power balance in region II is exceedingly simple,

$$
\Gamma^{S^{1, .1}}=0=\frac{c}{4}\left(S^{\mathrm{I}, \mathrm{II}}-S_{a n n}\langle f\rangle_{a n n}^{x}-S\langle f\rangle_{2 n d}^{x}\right) W_{x}^{\mathrm{I}}-\frac{c}{4} S^{\mathrm{I}, \mathrm{II}} W_{x}^{\mathrm{II}}=0
$$

The only energy source is the power incident on $S^{\mathrm{I}, 11}$ from region I reduced by that absorbed by the ring and second harmonic while all power flows back out through $S^{\text {I,II }}$ with no internal absorption or mode conversion. Using similar arguments, we find equations for the extraordinary mode in region III and for the ordinary mode throughout the plasma,

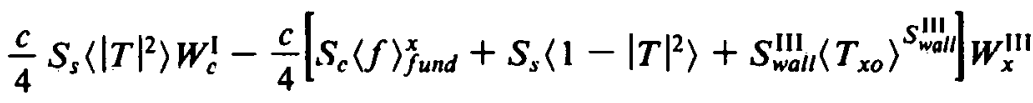

$$
\begin{aligned}
& +S_{w a l l}^{\mathrm{III}}\left\langle t_{o x}\right\rangle^{S_{w a l l}^{\prime \prime \prime}} W_{o}=-P_{x}^{\mathrm{III}} \\
& \frac{c}{4} S_{\text {wall }}^{1}\left\langle t_{x o}\right\rangle^{S_{w a l l}^{\prime}} W_{x}^{1}+\frac{c}{4} S_{w a l l}^{\mathrm{III}}\left\langle t_{x o}\right\rangle^{S_{\text {wall }}^{\prime \prime \prime}} W_{x}^{\mathrm{III}} \\
& -\frac{c}{4}\left[S_{w a l l}^{\mathrm{I}}\left\langle t_{o x}\right\rangle^{S_{w a l l}^{\mathrm{I}}}+S_{\text {wall }}^{\mathrm{III}}\left\langle t_{o x}\right\rangle^{S_{w a l l}^{\mathrm{III}}}+2 S_{c}\langle f\rangle_{\text {fund }}^{o}\right. \\
& \left.+2 S_{a n n}\langle f\rangle_{a n n}^{o}+2 S_{2 n d}\langle f\rangle_{2 n d}^{o}\right]=-P_{o}
\end{aligned}
$$

where $P_{o}$ and $P_{x}^{I I I}$ are the injected powers for ordinary mode and extraordinary mode, respectively, in region III. The factor of 2 associated with the absorption terms in Eq. (A.13) arises because both sides of the resonant zones are accessible to the ordinary mode.

After Eqs. (A.10)-(A.13) are solved for $W_{x}^{\mathrm{I}}, W_{x}^{\mathrm{II}}, W_{x}^{\mathrm{III}}$, and $W_{o}$, Eqs. (A.10, (A.3), and (A.6) can be used to find the power absorbed by each plasma component:

$$
\begin{aligned}
& P_{a n n}=\frac{c}{4} S_{a}\left[\langle f\rangle_{a n n}^{x}\left(W_{x}^{\mathrm{I}}+W_{x}^{\mathrm{II}}\right)+2\langle f\rangle_{a n n}^{o} W_{o}\right], \\
& P_{2 n d}=\frac{c}{4} S_{2 n d}\left[2\langle f\rangle_{2 n d}^{a n n}\left(W_{x}^{\mathrm{I}}+W_{x}^{\mathrm{II}}\right]+2\langle f\rangle_{2 n d}^{o} W_{o}\right], \\
& P_{\text {surf }}=\frac{c}{4} S_{s}\left[2\left\langle|A|^{2}\right\rangle W_{x}^{\mathrm{I}}+\left\langle 1-|T|^{2}\right\rangle W_{x}^{\mathrm{III}}\right], \\
& P_{\text {core }}=\frac{c}{4} S_{c}\left[S_{c}\left[\langle f\rangle_{f \text { und }}^{\mathrm{x}} W_{x}^{\mathrm{III}}+2\langle f\rangle_{f \text { fund }}^{o}\right] .\right.
\end{aligned}
$$

Here, we have maintained a distinction between the power deposited in the core plasma at the fundamental resonance $P_{\text {core }}$ and that deposited in the core plasma at the second harmonic $P_{2 n d}$. Since $P_{2 n d}$ is absorbed at the outer edge of the core plasma, additional discussion is necessary to decide whether some or all of it should be assigned to the surface plasma. 


\section{REFERENCES}

1. D. B. Batchelor and R. C. Goldfinger, Nucl. Fusion 20, 403 (1980).

2. D. B. Batchelor, R. C. Goldfinger, and H. Weitzner, IEEE Trans. Plasma Sci. PS-8, 78 (1980).

3. D. B. Batchelor and R. C. Goldfinger, RAYS: A Geometrical Optics Code for EBT (ORNL/TM-6844, Oak Ridge, 1982).

4. D. B. Batchelor, Nucl. Fusion 21, 1615 (1981).

5. D. B. Batchelor, R. C. Goldfinger, and H. Weitzner, "Electron Cyclotron Damping in Fully Relativistic Plasmas" (to be published).

6. D. B. Nelson and C. L. Hedrick, Nucl. Fusion 19, 235 (1979).

7. D. L. Hillis, private communication.

8. D. B. Batchelor, Plasma Physics 22, 41 (1980).

9. T. Uckan, L. A. Berry, D. L. Hillis, and R. K. Richards, Phys. Fluids 25, 1253 (1982).

10. N. A. Uckan and EBT Group, Plasma Phys. 25, 129 (1983).

11. G. R. Haste, in Hot Electron Ring Physics: Proceedings of the 2nd Workshop, San Diego, California, 1981, Vol. I, p. 23. 
ORNL/TM-8770

Dist. Category UC-20

INTERNAL DISTRIBUTION

1. F. W. Baity

2. J. K. Ballou

3-7. D. B. Batchelor

8. R. D. Burris

9. R. J. Colchin

10. W. A. Davis

11. H. O. Eason

12. J. C. Glowienka

13. R. C. Goldfinger

14. G. R. Haste

15. D. L. Hillis

16. Y-K. M. Peng

17. D. A. Rasmussen

18. R. K. Richards
19. J. A. Rome

20. M. J. Saltmarsh

21. J. Sheffield

22. D. A. Spong

23. N. A. Uckan

24. T. Uckan

25-26. . Laboratory Records Department

27. Laboratory Records, ORNL-RC

28. Central Research Library

29. Document Reference Section

30. ORNL Patent Office

31. Fusion Energy Division Library

32. Fusion Energy Division Reports Office

\section{EXTERNAL DISTRIBUTION}

33. Office of the Assistant Manager for Energy Research and Development, Department of Energy, Oak Ridge Operations, Box E, Oak Ridge, TN 37830

34. J. D. Callen, Department of Nuclear Engineering, University of Wisconsin, Madison, WI 53706

35. R. W. Conn, Department of Chemical, Nuclear, and Thermal Engineering, University of California, Lús Angeles, CA 90024

36. S. O. Dean, Director, Fusion Energy Development, Science Applications, Inc., 2 Professional Drive, Gaithersburg, MD 20760

37. H. K. Forsen, Bechtel Group, Inc., Research Engineering, P.O. Box 3965, San Francisco, CA 94105

38. R. W. Guuld, Department of Applied Physics, California Institute of Technology, Pasadena, CA 91125

39. D. G. McAlees, Exxon Nuclear Company, Inc., 777 106th Avenue, NE, Bellevue, WA 98009

40. P. J. Reardon, Princeton Plasma Physics Laboratory, P.O. Box 451, Princeton, NJ 08540

41. W. M. Stacey, Jr., School of Nuclear Engineering, Georgia Institute of Technology, Atlanta, GA 30332

42. G. A. Eliseev, I. V. Kurchatov Institute of Atomic Energy, P.O. Box 3402, 123182 Moscow, U.S.S.R.

43. V. A. Glukhikh, Scientific-Research Institute of Electro-Physical, Apparatus, 188631 Leningrad, U.S.S.R.

44. I. Spighel, Lebedev Physical Institute, Leninsky Prospect 53, 117924 Moscow, U.S.S.R.

45. D. D. Ryutov, Institute of Nuclear Physics, Siberian Branch of the Academy of Sciences of the U.S.S.R., Sovetskaya St. 5, 630090 Novosibirsk, U.S.S.R.

46. V. T. Tolok, Kharkov Physical-Technical Institute, Academical St. 1, 310108 Kharkov, U.S.S.R. 
47. R. Varma, Physical Research Laboratory, Navangpura, Ahmedabad, India

48. Bibliothek, Max-Planck Institut fur Plasmaphysik, D-8046 Garching bei Munchen, Federal Republic of Germany

49. Bibliothek, Institut fur Plasmaphysik, KFA, Postfach 1913, D-5170 Julich, Federal Republic of Germany

50. Bibliotheque, Centre de Recherches en Physique des Plasmas, 21 Avenue des Bain, 1007 Lausanne, Switzerland

51. Bibliotheque, Service du Confinement des Plasmas, CEA, B.P. 6, 92 Fontenay-aux-Roses (Seine), France

52. Documentation S.I.G.N., Department de la Physique du Plasma et de la Fusion Controlee, Centre d'Etudes Nucleaires, B.P. No. 85, Centre du Tri, 38041 Cedex, Grenoble, France

53. Library, Culham Laboratory, UKAEA, Abingdon, Oxon, OX14 3DB, England

54. Library, FOM Institut voor Plasma-Fysica, Rijnhuizen, Jutphaas, The Netherlands

55. Library, Institute of Physics, Academia Sinica, Beijing, Peoples Republic of China

56. Library, Institute of Plasma Physics, Nagoya University, Nagoya 64, Japan

57. Library, International Centre for Theoretical Physics, Trieste, Italy

58. Library, Laboratorio Gas Ionizzati, Frascati, Italy

59. Library, Plasma Physics Laboratory, Kyoto University, Gokasho Uji, Kyoto, Japan

60. Plasma Research Laboratory, Australian National University, P.O. Box 4, Canberra, A.C.T. 2000, Australia

61. Thermonuclear Library, Japan Atomic Energy Research Institute, Tokai, Naka, Ibaraki, Japan

62. J. F. Clarke, Associate Director for Fusion Energy, Office of Fusion Energy, Office of Energy Research, Mail Stop G-256, U.S. Department of Energy, Washington, DC 20545

63. J. F. Decker, Director, Division of Applied Plasma Physics, Office of Fusion Energy, Office of Energy Research, Mail Stop G-256, U.S. Department of Energy, Washington, DC 20545

64. D. B. Nelson, Fusion Theory and Computer Services Branch, Office of Fusion Energy, Office of Energy Research, Mail Stop G-256, U.S. Department of Energy, Washington, DC 20545

65. M. N. Rosenbluth, RLM 11.218, Institute for Fusion Studies, Uniiverisity of Texas, Austin, TX 78712

66. W. Sadowski, Fusion Theory and Computer Services Branch, Office of Fusion Energy, Officc of Energy Research, Mail Stop G-256, U.S. Department of Energy, Washington, DC 20545

67. A. Opdenaker, Reactor Systems and Applications Branch, Office of Fusion Energy, Office of Energy Research, Mail Stop G-256, U.S. Department of Energy, Washington, DC 20545

68. J. E. Baublitz, Reactor Systems and Applications Branch, Office of Fusion Energy, Office of Energy Research, Mail Stop G-256, U.S. Department of Energy, Washington, DC 20545

69. W. R. Ellis, Mirror Systems Branch, Office of Fusion Energy, Office of Energy Research, Mail Stop G-256, U.S. Department of Energy, Washington, DC 20545

70. J. M. Turner, Mirror Systems Branch, Office of Fusion Energy, Office of Energy Research, Mail Stop G-256, U.S. Department of Energy, Washington, DC 20545

71. T. V. George, Mirror Systems Branch, Office of Fusion Energy, Office of Energy Research, Mail Stop G-256, U.S. Department of Energy, Washington, DC 20545

72. Theory Department Read File, c/o D. W. Ross, Institute for Fusion Studies, University of Texas at Austin, Austin, TX 78712 
73. Theory Department Read File, c/o R. C. Davison, Director, Plasma Fusion Center, 167 Albany Street, Cambridge, MA 02139

74. Theory Department Read File, c/o F. W. Perkins, Princeton Plasma Physics Laboratory, P.O. Box 451, Princeton, NJ 08540

75. Theory Department Read File, c/o L. Kovrizhnykh, Lebedev Institute of Physics, Academy of Sciences, 53 Leninsky Prospect, Moscow, U.S.S.R. V312

76. Theory Department Read File, c/o B. B. Kadomtsev, I. V. Kurchatov Institute of Atomic Energy, P.O. Box 3402; Moscow, U.S.S.R. 123182

77. Theory Department Read File, c/o T. Kamimura, Institute of Plasma Physics, Nagoya University, Nagoya, Japan

78. Theory Department Read File, c/o C. Mercier, Euratom-CEA, Service de Recherches sur la Fusion Controlee, Fontenay-aux-Roses (Seine), France

79. Theory Department Read File, c/o T. E. Stringer, JET Joint Undertaking, Culham Laboratory, Abingdon, Oxon, OX14 3DB, England

80. Theory Department Read File, c/o K. Roberts, Culham Laboratory, Abingdon, Oxon, OX14 3DB, England

81. Theory Department Read File, c/o D. Biskamp, Max-Planck-Institut fur Plasmaphysik, D-8046 Garching bei Munchen, Federal Republic of Germany

82. Theory Department Read File, c/o T. Takeda, Japan Atomic Energy Research Institute, Tokai, Naka, Ibaraki, Japan

83. Theory Department Read File, c/o C. S. Liu, General Atomic Company, P.O. Box 81608, San Diego, CA 92138

84. Theory Department Read File, c/o L. D. Pearlstein, Lawrence Livermore National Laboratory, P.O. Box 808, Livermore, CA 94550

85. Theory Department Read File, c/o R. Gerwin, CTR Division, MS 640, Los Alamos National Laboratory, P.O. Box 1663, Los Alamos, NM 87545

86-192. Given distribution as shown in TID-4500, Magnetic Fusion Energy (Category Distribution UC-20) 